\title{
Anosov flows, growth rates on covers and group extensions of subshifts
}

\author{
Rhiannon Dougall ${ }^{1,2}$ - Richard Sharp ${ }^{3}$
}

Received: 29 April 2019 / Accepted: 11 August 2020 / Published online: 26 August 2020

(C) The Author(s) 2020

\begin{abstract}
The aim of this paper is to study growth properties of group extensions of hyperbolic dynamical systems, where we do not assume that the extension satisfies the symmetry conditions seen, for example, in the work of Stadlbauer on symmetric group extensions and of the authors on geodesic flows. Our main application is to growth rates of periodic orbits for covers of an Anosov flow: we reduce the problem of counting periodic orbits in an amenable cover $X$ to counting in a maximal abelian subcover $X^{\mathrm{ab}}$. In this way, we obtain an equivalence for the Gurevič entropy: $h(X)=h\left(X^{\mathrm{ab}}\right)$ if and only if the covering group is amenable. In addition, when we project the periodic orbits for amenable covers $X$ to the compact factor $M$, they equidistribute with respect to a natural equilibrium measure - in the case of the geodesic flow, the measure of maximal entropy.
\end{abstract}

$凶$ Rhiannon Dougall

R.Dougall@bristol.ac.uk

Richard Sharp

R.J.Sharp@warwick.ac.uk

1 School of Mathematics, University of Bristol, Bristol BS8 1UG, UK

2 Heilbronn Institute for Mathematical Research, Bristol, UK

3 Mathematics Institute, University of Warwick, Coventry CV4 7AL, UK 


\section{Introduction}

The aim of this paper is to study growth properties of group extensions of hyperbolic dynamical systems, where we do not assume that the extension satisfies the symmetry conditions imposed in [14] and [42], for example. Our main application is to growth rates of periodic orbits for covers of Anosov flows and we obtain generalisations of results previously known for geodesic flows over compact (or even convex co-compact) negatively curved manifolds $[14,35,42]$. We begin by describing these results.

Let $M$ be a compact smooth Riemannian manifold and let $\phi^{t}: M \rightarrow M$ be a transitive Anosov flow. Then $\phi^{t}$ has a countable set of periodic orbits $\mathcal{P}(\phi)$ and, for $\gamma \in \mathcal{P}(\phi)$, we write $l(\gamma)$ for its period. It is well-known that the growth rate of periodic orbits is given by the topological entropy of $\phi^{t}$; more precisely

$$
\lim _{T \rightarrow \infty} \frac{1}{T} \log \#\{\gamma \in \mathcal{P}(\phi): l(\gamma) \leq T\}=: h=h_{\text {top }}(\phi) .
$$

Now suppose that $X$ is a regular cover of $M$ with covering group $G$, i.e. $G$ acts freely and isometrically on $X$ such that $M=X / G$. Let $\phi_{X}^{t}: X \rightarrow X$ be the lifted flow, which we assume to be transitive. We will be interested in the growth of periodic orbits for $\phi_{X}^{t}$. If $G$ is finite then $\phi_{X}^{t}$ is also an Anosov flow and $h_{\text {top }}\left(\phi_{X}\right)=h_{\text {top }}(\phi)$. If $G$ is infinite the situation is more interesting. First, note that if $\phi_{X}^{t}$ has a periodic orbit $\gamma$ then the translates of $\gamma$ by the action of $G$ give infinitely many periodic orbits with the same period, so a naive definition of periodic orbit growth does not make sense. Rather, we follow the approach of [29] and, choosing an open, relatively compact set $W \subset X$, define

$$
h(X):=\limsup _{T \rightarrow \infty} \frac{1}{T} \log \#\left\{\gamma \in \mathcal{P}\left(\phi_{X}\right): l(\gamma) \leq T, \gamma \cap W \neq \varnothing\right\} .
$$

As the notation suggests, $h(X)$ is independent of the choice of $W$ (see Lemma 2.3). The above definition is analogous to that made by Gurevič for countable state Markov shifts [19] (see below) and it is natural to call $h(X)$ the Gurevič entropy of $\phi_{X}^{t}$. It is easy to see that $h(X) \leq h$.

Let us now restrict to the special case that $M=S V$ is the unit-tangent bundle over a compact manifold $V$ with negative sectional curvatures and that $\phi^{t}$ is the geodesic flow. (Notice that this flow admits a time-reversing involution $\iota: S V \rightarrow S V$ defined by $\iota(x, v)=(x,-v)$, where $x \in V$ and $v \in S_{x} V$.) Combining the results of [35] and [14], we have $h(X)=h$ if and only if $G$ is amenable. (Recall that $G$ is amenable if it has a Banach mean, that is, there is a $G$-invariant bounded linear functional $\mathfrak{M}: \ell^{\infty}(G, \mathbb{R}) \rightarrow \mathbb{R}$ such that, for $x \in \ell^{\infty}(G, \mathbb{R}), \inf _{g \in G} x(g) \leq \mathfrak{M}(x) \leq \sup _{g \in G} x(g)$.) This equivalence 
fails to hold for general Anosov flows - however, the results of this paper will say that, for an amenable cover, the counting is reduced to the maximal abelian subcover. This will be discussed in greater detail in Sect. 2 but we note at this point that if $Y$ is an abelian cover of $M$ then $h(Y)=h$ if and only if $\Phi_{\mu_{0}}^{Y}=0$, where $\Phi_{\mu_{0}}^{Y}$ is the (relative) winding cycle associated to the measure of maximal entropy for $\phi^{t}$. Our main result is that the correct comparison is between $h(X)$ and $h\left(X^{\mathrm{ab}}\right)$, where $X^{\mathrm{ab}}$ is the maximal abelian subcover of the cover $X \rightarrow M$ (so that the covering group for $X^{\mathrm{ab}} \rightarrow M$ is $G^{\mathrm{ab}}=G /[G: G]$, the abelianization of $G)$. Clearly, $h(X) \leq h\left(X^{\mathrm{ab}}\right)$ and we have the following theorem.

Theorem 1.1 We have $h(X)=h\left(X^{\mathrm{ab}}\right)$ if and only if $G$ is amenable.

An immediate consequence of this result and the characterisation of $h\left(X^{\mathrm{ab}}\right)$ in Proposition 2.4 is the following corollary, which expresses $h(X)$ in terms of measure-theoretic entropies of $\phi$-invariant measures. Let $\mathcal{M}(\phi)$ denote the set of $\phi$-invariant probability measures on $M$ and, for $\mu \in \mathcal{M}(\phi)$, let $h_{\phi}(\mu)$ denote the measure-theoretic entropy of $\phi$ with respect to $\mu$.

Corollary 1.2 We have $h(X)=\sup \left\{h_{\phi}(\mu): \mu \in \mathcal{M}(\phi), \Phi_{\mu}^{X^{\mathrm{ab}}}=0\right\}$ if and only if $G$ is amenable.

Readers more familiar with geodesic flows may wonder at this point, when is $\pi_{1}(M)$ non-amenable? In general it is very difficult to say anything about the topology of $M$ as the flow lines may not be efficient for measuring the fundamental group (for a discussion in dimension 3 see $[4,16]$ ). Nevertheless, our method is able to relate orbits and topology for transitive covers.

The Proof of Theorem 1.1 will use symbolic dynamics and, in particular, group extensions of subshifts of finite type. As part of the our approach we will obtain results in this setting which are of independent interest. (Some definitions are deferred until Sect. 3.) Let $\sigma: \Sigma \rightarrow \Sigma$ be a mixing subshift of finite type. We will write $h(\sigma)$ for the topological entropy of $\sigma$ and note that this is equal to the exponential growth rate of periodic points,

$$
h(\sigma)=\lim _{n \rightarrow \infty} \frac{1}{n} \log \#\left\{x \in \Sigma: \sigma^{n} x=x\right\} .
$$

For a countable group $G$ and a function $\psi: \Sigma \rightarrow G$, we consider the skewproduct dynamical system

$$
T_{\psi}: \Sigma \times G \rightarrow \Sigma \times G:(x, g) \mapsto(\sigma x, g \psi(x)) .
$$

We say that $T_{\psi}: \Sigma \times G \rightarrow \Sigma \times G$ is a $G$-extension of $\sigma: \Sigma \rightarrow \Sigma$. We will always assume that $T_{\psi}: \Sigma \times G \rightarrow \Sigma \times G$ is transitive. Let us consider 
the periodic points of $T_{\psi}$. Clearly, $T_{\psi}^{n}(x, g)=(x, g)$ if and only if $\sigma^{n} x=x$ and $\psi^{n}(x)=e$, the identity in $G$. If $G$ is infinite then $T_{\psi}^{n}$ has infinitely many periodic points of fixed period $n$; however, we can use the following definition, due to Gurevič [19], to obtain a growth rate. We say that the Gurevič entropy of $T_{\psi}$ is

$$
h_{\mathrm{Gur}}\left(T_{\psi}\right)=\limsup _{n \rightarrow \infty} \frac{1}{n} \log \#\left\{x \in \Sigma: \sigma^{n} x=x, \psi^{n}(x)=e\right\} .
$$

Clearly, $h_{\mathrm{Gur}}\left(T_{\psi}\right) \leq h(\sigma)$. However, it is easy to construct examples where $G$ is amenable but $h_{\text {Gur }}\left(T_{\psi}\right)<h(\sigma)$. For example, let $\Sigma=\{0,1,2\}^{\mathbb{Z}}, G=\mathbb{Z}$ and define $\psi$ by $\psi(x)=\psi\left(x_{0}\right)$ with $\psi(0)=\psi(1)=1$ and $\psi(2)=-1$. Then $T_{\psi}$ is transitive. By [32],

$$
h_{\mathrm{Gur}}\left(T_{\psi}\right)=\sup \left\{h_{\sigma}(m): m \in \mathcal{M}(\sigma), \int \psi d m=0\right\} .
$$

Now, the measure of maximal entropy $m_{0}$ for $\sigma: \Sigma \rightarrow \Sigma$ is the $(1 / 3,1 / 3,1 / 3)$-Bernoulli measure and, clearly, $\int \psi d m_{0}=1 / 3 \neq 0$. Thus, by the Variational Principle, $h_{\mathrm{Gur}}\left(T_{\psi}\right)<h(\sigma)$.

We shall show that, in fact, the natural comparison is between $h_{\mathrm{Gur}}\left(T_{\psi}\right)$ and $h_{\mathrm{Gur}}\left(T_{\psi^{\mathrm{ab}}}\right)$, where $T_{\psi^{\mathrm{ab}}}: \Sigma \times G^{\mathrm{ab}} \rightarrow \Sigma \times G^{\mathrm{ab}}$ is the induced $G^{\mathrm{ab}}{ }_{-}$ extension, where $G^{\mathrm{ab}}=G /[G, G]$ is the abelianization of $G$. More precisely, if $\pi: G \rightarrow G^{\mathrm{ab}}$ is the natural projection then $\psi^{\mathrm{ab}}=\pi \circ \psi$.

Theorem 1.3 If $T_{\psi}$ is transitive then $h_{\mathrm{Gur}}\left(T_{\psi}\right)=h_{\mathrm{Gur}}\left(T_{\psi \mathrm{ab}}\right)$ if and only if $G$ is amenable.

We now put these results into a broader context. This discussion will focus on more recent viewpoints, and on the role of symmetry, and so neglects to mention the spectral analogues of Brooks [7,8], whose work was a motivating factor in the recent developments of this field (for instance [9,10,13,14,42]).

We have already given the definition of an amenable group in terms of the existence of a Banach mean. We give an equivalent criterion of amenability due to Kesten [23], which is often taken as the definition for amenability. Throughout, let $p$ be a probability measure on $G$, which we will assume to be finitely supported and for the support to generate $G$. We say that $p$ is symmetric if $p(g)=p\left(g^{-1}\right)$ for all $g \in G$.

Kesten's theorem says that, for a symmetric probability on $G$, the decay of the probability to return to the identity is subexponential if and only if $G$ is amenable. More precisely, writing $\lambda(p)=\lim _{\sup } \operatorname{si\infty }_{n \rightarrow \infty}\left(p^{* n}(e)\right)^{1 / n}$, where $p^{* n}$ denotes the $n$th convolution of $p$, we have $\lambda(p)=1$ if and only if $G$ is amenable. This result has an application to counting for normal subgroups of 
non-elementary Gromov hyperbolic groups. Let $\Gamma$ be a non-elementary Gromov hyperbolic group and $\Gamma^{\prime}$ a normal subgroup. The uniform measure on the ball of radius $n$ in $\Gamma$ descends to a probability measure $p_{n}$ on $G=\Gamma / \Gamma^{\prime}$. It can be shown, for example in [9], that $\lim \sup _{n \rightarrow \infty} \log \lambda\left(p_{n}\right) \leq \delta_{\Gamma^{\prime}}-\delta_{\Gamma} \leq 0$; and so in the case that $G$ is amenable we have equality between the critical exponents $\delta_{\Gamma^{\prime}}, \delta_{\Gamma}$. (We also mention the more general approach of Roblin [35] who uses the Banach mean property to prove the result only for normal subgroups $\Gamma^{\prime}$ of $\Gamma$ a non-elementary discrete group of isometries of a simply connected negatively curved manifold - notably there is no compactness assumption on $\Gamma$.) Because of this avenue of approximating by a sequence of probability measures $p_{n}$, the community has sometimes referred to statements " $G$ amenable implies ..." as "the easy direction". However, it is clear that the aforementioned application crucially used the existence of a natural family of symmetric probability measures. As we will see from the literature reviewed in Sect. 2.3, extending such orbital counting results beyond the inherent symmetry of the isometric actions to Anosov flows is highly non-trivial.

The case that $G$ is non-amenable is more robust in the absence of symmetry. In the case of random walks, it can happen that $G$ is amenable, and $\lambda(p)<1$. The Kesten criterion was generalised by Day [11] to a criterion on the $\ell^{2}(G)$ spectral radius $\operatorname{spr}\left(M_{p}\right)$ of the random walk operator $M_{p} f(x)=\sum_{g} p(g) f(x g)$. In this way, $G$ is amenable if and only if $\operatorname{spr}\left(M_{p}\right)=1$. Observe that $\lambda(p) \leq \operatorname{spr}\left(M_{p}\right)$ and so one direction of the Kesten result is contained in this. In the setting of subshifts of finite type, it is natural to consider the transfer operator $\mathcal{L}_{0}$ (for a full exposition see Sect. 4). Stadlbauer [42] showed that $\operatorname{spr}\left(\mathcal{L}_{0}\right)<\exp (h(\sigma))$ if $G$ non-amenable; and Jaerisch [20] showed the converse. Analogous to the case of random walks, the growth quantity we wish to estimate for subshifts of finite type satisfies $\exp \left(h_{\text {Gur }}\left(T_{\psi}\right)\right) \leq \operatorname{spr}\left(\mathcal{L}_{0}\right)$.

We conclude the introduction by outlining the contents of the rest of the paper. In Sect. 2 we introduce Anosov flows and their lift to covers. In particular, we give an account of results about growth of periodic orbits for abelian covers. In Sect. 3, we discuss subshifts of finite type and introduce the notion of Gurevič pressure. In Sect. 4, we discuss Gurevič entropy for group extensions of subshifts of finite type and prove one direction in Theorem 1.3. The Proof of Theorem 1.3 is completed in Sect. 5, which contains the key to obtaining results in the absence of symmetry. In Sect. 6, we return to Anosov flows and discuss how they may be coded in terms of subshifts of finite type and Sect. 7 extends this to covers and group extensions. In Sect. 8, we complete the Proof of Theorem 1.1. Finally, in Sect. 9, we state and proof a number of equidistribution results. 


\section{Anosov flows}

\subsection{Anosov flows, periodic orbits and pressure}

Let $\phi^{t}: M \rightarrow M$ be an Anosov flow, i.e. that the tangent bundle has a continuous $D \phi$-invariant splitting $T M=E^{0} \oplus E^{s} \oplus E^{u}$, where $E^{0}$ is the one-dimensional bundle tangent to the flow and where there exist constants $C, \lambda>0$ such that

(1) $\left\|D \phi^{t} v\right\| \leq C e^{-\lambda t}\|v\|$, for all $v \in E^{s}$ and $t>0$;

(2) $\left\|D \phi^{-t} v\right\| \leq C e^{-\lambda t}\|v\|$, for all $v \in E^{u}$ and $t>0$.

In addition, we assume that $\phi^{t}: M \rightarrow M$ is transitive and weak mixing.

For some intuition behind Anosov flows, one should note the following constructions (and we will make use of them soon). An $\epsilon$-pseudo-orbit for $\phi^{t}$ is a path $\tau: I \rightarrow M$ (where $I$ is an interval) so that for all $t, t+\delta \in I,|\delta|<1$,

$$
d\left(\tau(t+\delta), \phi^{\delta}(\tau(t))\right)<\epsilon .
$$

We say that $\tau: \mathbb{R} \rightarrow M$ is a periodic $\epsilon$-pseudo-orbit if it is a periodic map of $\mathbb{R}$, and satisfies $d\left(\tau(t+\delta), \phi^{\delta}(\tau(t))\right)<\epsilon$ for all $t, t+\delta \in \mathbb{R},|\delta|<1$.

Lemma 2.1 (Anosov Closing Lemma, [17], Theorem 5.3.10) Let $\Lambda$ be a hyperbolic set for a flow $\phi^{t}$. Then there exists a neighbourhood $U$ of $\Lambda$ and numbers $\epsilon_{0}, L>0$ such that for $\epsilon \leq \epsilon_{0}$, any periodic $\epsilon$-pseudo-orbit is $L \epsilon$ shadowed by a unique periodic orbit for $\phi^{t}$.

Write $\mathcal{P}(\phi)$ for the set of periodic orbits of $\phi$ and, for $\gamma \in \mathcal{P}(\phi)$, write $l(\gamma)$ for its period. The limit

$$
h=\lim _{T \rightarrow \infty} \frac{1}{T} \log \#\{\gamma \in \mathcal{P}(\phi): l(\gamma) \leq T\}
$$

exists and is positive, and $h$ is also equal to the topological entropy of $\phi$. We also have the variational principle

$$
h=\sup \left\{h_{\phi}(\mu): \mu \in \mathcal{M}(\phi)\right\},
$$

where $h_{\phi}(\mu)$ denotes the measure-theoretic entropy of $\phi$ with respect to $\mu$, and $\mathcal{M}(\phi)$ is the collection of $\phi$-invariant Borel probability measures on $M$. The supremum is attained at a unique measure $\mu_{0}$, called the measure of maximal entropy for $\phi$. We also have a weighted version of this set-up. For a continuous function $F: M \rightarrow \mathbb{R}$, we can define the pressure $P(F, \phi)$ by

$$
P(F, \phi)=\lim _{T \rightarrow \infty} \frac{1}{T} \log \sum_{\substack{\gamma \in \mathcal{P}(\phi): \\ l(\gamma) \leq T}} \exp \int_{\gamma} F d t,
$$


where

$$
\int_{\gamma} F:=\int_{0}^{l(\gamma)} F\left(\phi_{t} x_{\gamma}\right) d t
$$

with $x_{\gamma} \in \gamma$. In this case, we also have a variational principle,

$$
P(F, \phi)=\sup \left\{h_{\phi}(\mu)+\int_{M} F d \mu: \mu \in \mathcal{M}(\phi)\right\} .
$$

The supremum is attained by a unique measure $\mu_{F}$ which is ergodic; we call $\mu_{F}$ the equilibrium state for $F$. In Sect. 9, we will use the following lemma. (The proofs are completely analogous to those Theorem 8.2 and Theorem 9.12 of [44], which deal with a single transformation. In particular, the first statement follows from the fact that the flow is expansive. Once we have established upper semi-continuity, rearranging the variational principle above into this form follows the same argument as the proof of Theorem 9.12 of [44].)

Lemma 2.2 The map $\mathcal{M}(\phi) \rightarrow \mathbb{R}: \mu \mapsto h_{\phi}(\mu)$ is upper semi-continuous and

$$
h_{\phi}(\mu)=\inf \left\{P(F, \phi)-\int_{M} F d \mu: F \in C(M, \mathbb{R})\right\} .
$$

For Hölder continuous functions $F, G: M \rightarrow \mathbb{R}$, the function $\mathbb{R} \rightarrow \mathbb{R}$ : $t \mapsto P(F+t G)$ is real-analytic and

$$
\left.\frac{d P(F+t G)}{d t}\right|_{t=0}=\int_{M} G d \mu_{F} .
$$

\subsection{Covers}

Suppose that $X$ is a regular cover of $M$ with covering group $G$. Let $\phi_{X}^{t}$ : $X \rightarrow X$ be the lift of $\phi$ to $X$. In particular, the $G$ action commutes with $\phi_{X}^{t}$. Write $\mathcal{P}\left(\phi_{X}\right)$ for the set of periodic orbits of $\phi_{X}$ and, as above, write $l(\gamma)$ for the period of the periodic orbit $\gamma$. Fix an open set $W$ in $M$ whose closure is compact. Write

$$
\pi_{X}(T, W)=\#\left\{\gamma \in \mathcal{P}\left(\phi_{X}\right): l(\gamma) \leq T \text { and } \gamma \cap W \neq \varnothing\right\}
$$

and

$$
h(X)=\limsup _{T \rightarrow \infty} \frac{1}{T} \log \pi_{X}(T, W)
$$


for the exponential growth rate of $\pi_{X}(T, W)$.

We also introduce another counting function associated to the cover $X$. Write $\Pi_{X}(T)$ for the cardinality of the set of periodic orbits for $\phi$ in $M$ of period at most $T$, and which lift to a periodic orbit for $\phi_{X}$.

We have the following lemma.

Lemma 2.3 The value of $h(X)$ is independent of the choice of $W$. In addition,

$$
h(X)=\limsup _{T \rightarrow \infty} \frac{1}{T} \log \Pi_{X}(T)
$$

Proof Let us write $h(X, W)=\lim \sup _{T \rightarrow \infty} \frac{1}{T} \log \pi_{X}(T, W)$ to emphasise the choice of $W$. We begin by showing that $h(X, W)$ is independent of the choice of $W$.

First, we observe that it is sufficient to consider "small" $W$. To see this, lift our preferred metric on $M$ to $X$. Since the closure of $W$ is compact, it has bounded diameter $R$. For every $\epsilon>0$ we can find a finite family of open sets of diameter less than $\epsilon$ that cover $W$. Then it is easy to see that there is $W_{\epsilon}$ of diameter less than $\epsilon$ with $h(X, W) \leq h\left(X, W_{\epsilon}\right)$. On the other hand, since $W$ is open it must contain an open set $W_{\delta}$ of diameter less than $\delta$ for all sufficiently small $\delta$. In this way we have $h\left(X, W_{\delta}\right) \leq h(X, W) \leq h\left(X, W_{\epsilon}\right)$.

Let $W_{1}, W_{2}$ be two sets of sufficiently small diameter diam $\left(W_{1}\right)$, diam $\left(W_{2}\right)<$ $\epsilon_{0}$, where $\epsilon_{0}$ is given by the Anosov Closing Lemma (Lemma 2.1). We will also assume that $\epsilon_{0}$ is sufficiently small that an open ball in $M$ of this diameter is simply connected.

We want to show that $h\left(X, W_{1}\right) \leq h\left(X, W_{2}\right)$.

As $W_{2}$ is open it contains a ball $B_{2}$ of radius some $\delta$ and a $\lambda \delta$ sub-ball, where $\lambda$ is chosen so that $\lambda \delta+L \lambda \delta<\delta$ and $L$ is also given by the Anosov Closing Lemma. Write $\delta_{2}=\lambda \delta$ (and note that $\delta_{2} \leq \epsilon_{0}$ ). Cover $W_{1}$ by finitely many $\delta_{2}$ balls $B_{1}^{i}$ (where $i$ runs over some finite indexing set). For each $i$, fix an orbit $c_{i}^{1}$ from $B_{1}^{i} \cap W_{1}$ to $B_{2}$. Fix orbits $c_{i}^{2}$ from $W_{2}$ to $B_{1}^{i} \cap W_{1}$. This is possible by transitivity. Let $\tau_{1}$ be a periodic $\phi_{X}$-orbit intersecting $W_{1}$ and write $T_{1}$ for its period. There is some $i$ so that $\tau_{1}$ is $\delta_{2}$ close to $c_{i}^{1}$ and $c_{i}^{2}$. Then $c_{i}^{1} \tau_{1} c_{i}^{2}$ is a $\delta_{2}$-pseudo-orbit through $B_{2}$.

Now consider the projection of $c_{i}^{1} \tau_{1} c_{i}^{2}$ to $M$. By the Anosov Closing Lemma, this is $L \delta_{2}$-shadowed by a unique periodic orbit $\gamma_{0}$. Since $L \delta_{2}<\epsilon_{0}, \gamma_{0}$ lifts to a $\phi_{X}$-periodic orbit $\gamma$ on $X$ which $L \delta_{2}$ shadows $c_{i}^{1} \tau_{1} c_{i}^{2}$. Unpicking the value of $\delta_{2}$, gives that $\gamma$ passes within distance $L \lambda \delta$ of the $\lambda \delta$ sub-ball. Since $\lambda \delta+L \lambda \delta<\delta$, we conclude that $\gamma$ passes through $B_{2}$, and therefore $W_{2}$. In addition, it is clear (for $T_{1}$ sufficiently large) that the period $T_{\gamma}$ of $\gamma$ is bounded by $\left|T_{\gamma}-T_{1}\right| \leq c_{i}^{1}+c_{i}^{2}+L \delta_{2}$. 
We have thus created a map from the set of $\phi_{X}$-periodic orbits intersecting $W_{1}$ to the set of $\phi_{X}$-periodic orbits intersecting $W_{2}$-periodic orbits, that distorts periods by an additive constant. Observe that when the period of $\tau_{1}$ is sufficiently large, the mapping is an injection (otherwise we would have distinct periodic orbits shadowing each other).

We have concluded that $h\left(X, W_{1}\right) \leq h\left(X, W_{2}\right)$. Since $W_{1}, W_{2}$ were arbitrary, this gives $h\left(X, W_{1}\right)=h\left(X, W_{2}\right)$ for all $W_{1}, W_{2}$, as required.

We finish be showing the final part of the lemma. In general, it is clear that

$$
h(X) \geq \limsup _{T \rightarrow \infty} \frac{1}{T} \log \Pi_{X}(T)
$$

(since we can choose $W$ to be a fundamental domain for the covering and so each $\gamma$ counted by $\Pi_{X}(T)$ will have at least one left intersecting $W$ ). To see the reverse inequality, we simply choose $W$ to be a small ball in $X$. Let

$$
C=\inf \left\{t>0: \exists x \in W \text { such that } \phi_{X}^{t} x \in G W \backslash W\right\},
$$

then $C>0$.

Let $\gamma$ be a $\phi_{X}$-periodic orbit of period $\leq T$ intersecting $W$, and let $\gamma_{0}$ be its projection to $M$. Fixing $\gamma_{0}$, there are at most $T / C$ such $\gamma$. In this way we deduce that $\Pi_{X}(T) \geq(C / T) \pi_{X}(T, W)$, and so $h(X)=$ $\lim \sup _{T \rightarrow \infty} T^{-1} \log \Pi_{X}(T)$.

\subsection{Abelian covers, relative winding cycles and counting}

We will now specialise to abelian covers of $M$; to emphasise this distinction, we shall denote the covering space by $Y$. Let $Y$ be a regular cover of $M$, with abelian covering group $G$. (Since $G$ is abelian, we will use 0 to denote its identity element.) We begin by defining winding cycles relative to this cover. First note that we may write $G=A \oplus F$, where $A$ is a free abelian group of rank $a \geq 0$ and $F$ is the finite subgroup of torsion elements. If $a=0$ then the lifted flow $\phi_{Y}^{t}: Y \rightarrow Y$ is also an Anosov flow and it has topological entropy equal to $h$, so there is nothing interesting to say in this case. We therefore suppose that $a \geq 1$. It is best to view $A$ as a free $\mathbb{Z}$-module.

The cover $Y$ is itself covered by the universal abelian cover $\bar{Y}$ of $M$ and there is a natural surjective homomorphism from $H_{1}(M, \mathbb{Z})$ to $G$ which (by factoring out $F$ ) induces a surjective module homomorphism $\alpha: H \rightarrow A$, where $H=H_{1}(M, \mathbb{Z}) /$ torsion is a free $\mathbb{Z}$-module of rank $b \geq a$. (Of course, $H$ is also identified as an integral lattice in $H_{1}(M, \mathbb{R})$.) We may then write $H=A \oplus \operatorname{ker} \alpha$. 
Let $c_{1}, \ldots, c_{a}$ be a basis for $A$ (which we may identify with elements of $H_{1}(M, \mathbb{R})$ ) and (if $b>a$ ) let $c_{a+1}, \ldots, c_{b} \in \operatorname{ker} \alpha$ extend this to a basis for $H$. Then $\left\{c_{1}, \ldots, c_{a}\right\}$ spans a vector subspace of $H_{1}(M, \mathbb{Z})$ which may be identified with $A \otimes_{\mathbb{Z}} \mathbb{R}$. We shall denote this vector space by $\Gamma(M, Y)$.

Now let $\omega_{1}, \ldots, \omega_{b}$ be closed 1-forms on $M$ representing cohomology classes $\left[\omega_{1}\right], \ldots,\left[\omega_{b}\right]$ in $H^{1}(M, \mathbb{R})$ which are dual to $c_{1}, \ldots, c_{b}$, i.e.

$$
\left\langle\left[\omega_{i}\right], c_{j}\right\rangle:=\int_{c_{j}} \omega_{i}=\delta_{i j} .
$$

(Here, $\delta_{i j}$ denotes the Kronecker symbol.) We can identify the span of $\left\{\left[\omega_{1}\right], \ldots,\left[\omega_{a}\right]\right\}$ with the dual space of $\Gamma(M, Y)$, which we denote by $\Gamma(M, Y)^{*}$. (If $Y=\bar{Y}$ then $\Gamma(M, Y)=H_{1}(M, \mathbb{R})$ and $\Gamma(M, Y)^{*}=$ $H^{1}(M, \mathbb{R})$.)

Now consider a measure $\mu \in \mathcal{M}(\phi)$. We define an element $\Phi_{\mu}^{Y} \in \Gamma(M, Y)$, called the winding cycle of $\mu$ relative to the cover $Y$, by its action on the dual space $\Gamma(M, Y)^{*}$. For $\left[\omega_{i}\right], i=1, \ldots, a$, we define

$$
\left\langle\Phi_{\mu}^{Y},\left[\omega_{i}\right]\right\rangle=\int_{M} \omega_{i}\left(\mathcal{X}_{\phi}\right) d \mu
$$

and extended by linearity, where $\mathcal{X}_{\phi}$ is the vector field generating $\phi$. (If $d f$ is an exact 1-form then $d f\left(\mathcal{X}_{\phi}\right)$ is the derivative of $f$ in the flow direction, i.e. $d f\left(\mathcal{X}_{\phi}\right)(x)=\lim _{t \rightarrow 0+} t^{-1}\left(f\left(\phi^{t} x\right)-f(x)\right)$. Hence, since $\mu$ is $\phi$-invariant, $\int_{M} d f\left(\mathcal{X}_{\phi}\right) d \mu=0$, so $\Phi_{\mu}^{Y}$ is well-defined.)

Associated to each periodic orbit $\gamma \in \mathcal{P}(\phi)$, there is an element $[\gamma] \in G$, defined as follows. Let $\gamma=\left\{\phi^{t} x: 0 \leq t \leq l(\gamma)\right\}$ and let $\tilde{x}$ be a lift of $x$ to $Y$. Then $\phi_{Y}^{l(\gamma)} \tilde{x}=g \widetilde{x}$, for some $g \in G$. Since $G$ is abelian, $g$ is independent of the choice of lift and we define $[\gamma]=g$. Notice that $\gamma$ lifts to a periodic orbit if and only if $[\gamma]=0$. We say that $\phi^{t}: M \rightarrow M$ is $Y$-full if $\{[\gamma]: \gamma \in \mathcal{P}(\phi)\}=G$. (This generalises the notion of homologically full, introduced in [39], for the case where $Y$ is the universal abelian cover of $M$.) We will show in Sect. 6 that if $\phi_{Y}^{t}: Y \rightarrow Y$ is transitive then $\phi$ is $Y$-full.

The counting function $\Pi_{Y}(T)$ introduced above may be written as $\Pi_{Y}(T)=$ $\#\{\gamma \in \mathcal{P}(\phi): l(\gamma) \leq T,[\gamma]=0\}$. The following result was proved in [39] in the case where $Y$ is the universal abelian cover and $G=H_{1}(M, \mathbb{Z})$ but the proof immediately extends to arbitrary abelian covers. The results in [39] are also phrased in terms of prime periodic orbits but it is easy to see that the number of non-prime periodic $\phi$-orbits that left to a periodic orbit on $Y$ is of order $O\left(T e^{h(Y) T / 2}\right.$ ) and so does not affect the asymptotic (see Lemma 2.8 below).

Proposition 2.4 (Sharp [39]) The following statements are equivalent: 
(i) the set $\left\{[\gamma]_{\mathrm{TF}}: \gamma \in \mathcal{P}(\phi)\right\}$ is not contained in a closed half-space of $\mathbb{R}^{a}$, where $[\gamma]_{\mathrm{TF}} \in \mathbb{R}^{a}$ is the torsion-free part of $[\gamma]$;

(ii) $\phi: M \rightarrow M$ is $Y$-full;

(iii) there exist a constant $C>0$ such that

$$
\Pi_{Y}(T) \sim C \frac{e^{h(Y) T}}{T^{1+a / 2}}, \text { as } T \rightarrow \infty .
$$

Furthermore,

$$
h(Y)=\sup \left\{h(\mu): \mu \in \mathcal{M}(\phi), \Phi_{\mu}^{Y}=0\right\} .
$$

In view of the uniqueness of the measure of maximal entropy, we immediately have the following corollary.

Corollary 2.5 We have $h(Y)=h$ if and only if $\Phi_{\mu_{0}}^{Y}=0$, where $\mu_{0}$ is the measure of maximal entropy for $\phi$.

Remark 2.6 (i) A similar asymptotic holds for $\#\{\gamma \in \mathcal{P}(\phi): l(\gamma) \leq$ $T,[\gamma]=\alpha\}$ for any $\alpha \in G$, the only modification being that the constant $C$ is changed to $C e^{\left\langle\xi, \alpha^{\prime}\right\rangle}$, where $\alpha^{\prime}$ is the torsion-free part of $\alpha$ and $\xi$ is a certain cohomology class in $H^{1}(M, \mathbb{R})$. In fact, $\xi=0$ if and only if $\Phi_{\mu_{0}}^{Y}=0$.

(ii) Earlier results were obtained for geodesic flows over compact negatively curved manifolds: Phillips and Sarnak [30] (constant curvature manifolds), Katsuda and Sunada [21] (constant curvature surfaces), Lalley [26] and Pollicott [31] (variable curvature surfaces). All these results exploited the time-reversal symmetry of the geodesic flow. The extension to Anosov flows was made by Katsuda and Sunada [22] under the assumption that the winding cycle for the measure of maximal entropy vanishes. Results giving more detailed information about the asymptotic behaviour are contained in $[1,25,33]$ and [40].

We can relate the growth rate $h(Y)$ to pressure in the following way [39]. For each $i=1, \ldots, a$, define a Hölder continuous function $\Psi_{i}: M \rightarrow \mathbb{R}$ by $\Psi_{i}=\omega_{i}\left(\mathcal{X}_{\phi}\right)$, where $\omega_{i}$ and $\mathcal{X}_{\phi}$ are as above. Now write $\Psi=\left(\Psi_{1}, \ldots, \Psi_{a}\right)$ and define $\beta: \mathbb{R}^{a} \rightarrow \mathbb{R}$ by

$$
\beta(w)=P(\langle w, \Psi\rangle, \phi),
$$

where $w=\left(w_{1}, \ldots, w_{a}\right)$ and $\langle w, \Psi\rangle=\sum_{i=1}^{a} w_{i} \Psi_{i}$. If $\phi$ is $Y$-full then the function $\beta$ is strictly convex and there exists a unique $\xi \in \mathbb{R}^{a}$ for which $\nabla \beta(\xi)=0$. We then have

$$
h(Y)=\beta(\xi)=h_{\phi}(\mu\langle\xi, \Psi\rangle) .
$$


Let us summarise this in a lemma.

Lemma 2.7 (Sharp [39]) If $\phi^{t}: M \rightarrow M$ is $Y$-full then there exists a unique $\xi \in \mathbb{R}^{a}$ such that

$$
h(Y)=\beta(\xi)=h_{\phi}\left(\mu_{\langle\xi, \Psi\rangle}\right) .
$$

Another characterisation of $h(Y)$ is as the abscissa of convergence of the series

$$
\sum_{\substack{\gamma \in \mathcal{P}(\phi): \\[\gamma]=0}} e^{-s l(\gamma)}
$$

Later, it will be convenient to replace this series with a modified version. We introduce some notation. Let $\mathcal{P}(\phi)^{\prime}$ denote the set of prime periodic orbits. For $\gamma \in \mathcal{P}(\phi)$, we define $\Lambda(\gamma)$ as follows. Any we may write $\gamma$ as $\gamma=\gamma_{0}^{m}$, where $\gamma_{0}$ is a prime periodic orbit and $m \geq 1$. Then $\Lambda(\gamma)=l\left(\gamma_{0}\right)$. We have the following lemma.

\section{Lemma 2.8 The series}

$$
\sum_{\substack{\gamma \in \mathcal{P}(\phi)^{\prime}: \\[\gamma]=0}} e^{-s l(\gamma)} \text { and } \sum_{\substack{\gamma \in \mathcal{P}(\phi): \\[\gamma]=0}} \frac{\Lambda(\gamma)}{l(\gamma)} e^{-s l(\gamma)}
$$

each have abscissa of convergence equal to $h(Y)$.

Proof First we note that

$$
\sum_{\substack{\gamma \in \mathcal{P}(\phi): \\[\gamma]=0}} e^{-s l(\gamma)}-\sum_{\substack{\gamma \in \mathcal{P}(\phi)^{\prime}: \\[\gamma]=0}} e^{-s l(\gamma)}=\sum_{m=2}^{\infty} \sum_{\substack{\gamma \in \mathcal{P}(\phi)^{\prime}: \\\left[\gamma^{m}\right]=0}} e^{-s m l(\gamma)}
$$

The abscissa of convergence of the Right Hand Side can be bounded by

$$
\begin{aligned}
\lim _{T \rightarrow \infty} \frac{1}{T} \log \sum_{m=2}^{\infty} \sum_{\substack{\gamma \in \mathcal{P}(\phi)^{\prime} \cdot \\
m l(\gamma) \leq T,\left[\gamma^{m}\right]=0}} 1 & =\lim _{T \rightarrow \infty} \frac{1}{T} \log \sum_{m=2}^{\infty} \sum_{\substack{\gamma \in \mathcal{P}(\phi)^{\prime}: \\
m l(\gamma) \leq T,\left[\gamma^{m}\right]=0}} e^{\left\langle\xi,\left[\gamma^{m}\right] \mathrm{TF}\right\rangle} \\
& \leq \lim _{T \rightarrow \infty} \frac{1}{T} \log \sum_{m=2}^{\infty} \sum_{\substack{\gamma \in \mathcal{P}(\phi)^{\prime}: \\
m l(\gamma) \leq T}} e^{\left\langle\xi,\left[\gamma^{m}\right] \mathrm{TF}\right\rangle}
\end{aligned}
$$




$$
\begin{aligned}
& \leq \lim _{T \rightarrow \infty} \frac{1}{T} \log \sum_{m=2}^{\left[T / l_{0}\right]} \sum_{\substack{\gamma \in \mathcal{P}(\phi): \\
l(\gamma) \leq T / 2}} e^{\left\langle\xi,\left[\gamma^{m}\right]_{\mathrm{TF}}\right\rangle} \\
& =P(\langle\xi, \Psi\rangle) / 2=h(Y) / 2,
\end{aligned}
$$

where $l_{0}$ denotes the period of the shortest orbit in $\mathcal{P}(\phi)$. The second statement follows from a similar argument.

As a part of our approach to Theorem 1.1 will capture information about an Anosov flow $\phi^{t}: M \rightarrow M$ and its lifts in terms of symbolic dynamical systems: subshifts of finite type and their skew-product extensions. We will introduce these systems in the next section and then go on to discuss their relation to Anosov flows in Sect. 6.

\section{Subshifts of finite type and group extensions}

In this section we will define countable state Markov shifts and discuss some of their properties. Basic definitions and results are taken from chapter 7 of [24]. Let us emphasise that throughout we have the hypothesis that our Markov shifts are locally compact (this excludes examples such as the infinite full shift and the renewal shift). We shall be particularly concerned with finite state shifts and skew product extensions of these by a countable group.

Let $S$ be a countable set, called the alphabet, and let $A$ be a matrix, called the transition matrix, indexed by $S \times S$ with entries zero or one. We then define the space

$$
\Sigma^{+}=\Sigma_{A}^{+}=\left\{x=\left(x_{n}\right)_{n=0}^{\infty} \in S^{\mathbb{Z}^{+}}: A\left(x_{n}, x_{n+1}\right)=1 \forall n \in \mathbb{Z}^{+}\right\},
$$

with the product topology induced by the discrete topology on $S$. This topology is compatible with the metric $d(x, y)=2^{-n(x, y)}$, where

$$
n(x, y)=\inf \left\{n: x_{n} \neq y_{n}\right\},
$$

with $n(x, y)=\infty$ if $x=y$. If $S$ is finite then $\Sigma^{+}$is compact. We say that $A$ is locally finite if all its row and column sums are finite. Then $\Sigma^{+}$is locally compact if and only if $A$ is locally finite. (The skew product extensions we consider have this latter property.)

We define the (one-sided) countable state topological Markov shift $\sigma$ : $\Sigma^{+} \rightarrow \Sigma^{+}$by $(\sigma x)_{n}=x_{n+1}$. This is a continuous map. We will say that $\sigma$ is topologically transitive if it has a dense orbit and topologically mixing if, given non-empty open sets $U, V \subset \Sigma^{+}$, there exists $N \geq 0$ such that $\sigma^{-n}(U) \cap V \neq \varnothing$ for all $n \geq N$. We say that the matrix $A$ is irreducible if, 
for each $(i, j) \in S \times S$, there exists $n=n(i, j) \geq 1$ such that $A^{n}(i, j)>0$. For $A$ irreducible, set $p \geq 1$ to be the greatest common divisor of periods of periodic orbits $\sigma: \Sigma^{+} \rightarrow \Sigma^{+}$; this $p$ is called the period of $A$. We say that $A$ is aperiodic if $p=1$ or, equivalently, if there exists $n \geq 1$ such that $A^{n}$ has all entries positive. Suppose that $A$ is locally finite. Then $\sigma: \Sigma^{+} \rightarrow \Sigma^{+}$is topologically transitive if and only if $A$ is irreducible and $\sigma: \Sigma^{+} \rightarrow \Sigma^{+}$is topologically mixing if and only if $A$ is aperiodic.

Suppose that $A$ is irreducible but not aperiodic and fix $i \in S$. Then we may partition $S$ into sets $S_{l}, l=0, \ldots, p-1$, defined by

$$
S_{l}=\left\{j: A^{n p+l}(i, j)>0 \text { for some } n \geq 1\right\} .
$$

(This partition is independent of the choice of $i$.) For each $l$, let $A_{l}$ denote the restriction of $A$ to $S_{l} \times S_{l}$; then $\sigma: \Sigma_{A_{l}}^{+} \rightarrow \Sigma_{A_{l+1}}^{+}(\bmod p)$ and $A_{l}^{p}$ is aperiodic.

We say that an $n$-tuple $w=\left(w_{0}, \ldots, w_{n-1}\right) \in S^{n}$ is an allowed word of length $n$ if $A\left(w_{j}, w_{j+1}\right)=1$ for $j=0, \ldots, n-2$. We will write $\mathcal{W}^{n}$ for the set of allowed words of length $n$. If $w \in \mathcal{W}^{n}$ then we define the associated length $n$ cylinder set $[w]$ by

$$
[w]=\left\{x \in \Sigma_{A}^{+}: x_{j}=w_{j}, j=0, \ldots, n-1\right\} .
$$

For a function $f: \Sigma^{+} \rightarrow \mathbb{R}$, set

$$
V_{n}(f)=\sup \left\{|f(x)-f(y)|: x_{j}=y_{j}, j=0, \ldots, n-1\right\} .
$$

We say that $f$ is locally Hölder continuous if there exist $\alpha>0$ and $C \geq 0$ such that, for all $n \geq 1, V_{n}(f) \leq C 2^{-n \alpha}$. (There is no requirement on $V_{0}(f)$ and a locally Hölder $f$ may be unbounded.) The minimal possible $C$ is called the $\alpha$-Hölder seminorm

Suppose that $\sigma: \Sigma^{+} \rightarrow \Sigma^{+}$is topologically transitive and let $f: \Sigma^{+} \rightarrow \mathbb{R}$ be a locally Hölder continuous function. Following Sarig [37], we define the Gurevič pressure, $P_{\mathrm{Gur}}(f, \sigma)$, of $f$ to be

$$
P_{\text {Gur }}(f, \sigma)=\limsup _{n \rightarrow \infty} \frac{1}{n} \log \sum_{\begin{array}{c}
\sigma^{n} x=x \\
x_{0}=a
\end{array}} e^{f^{n}(x)},
$$

where $a \in S$. (The definition is independent of the choice of $a$.)

Remark 3.1 In [37], Sarig gives this definition in the case where $\sigma: \Sigma^{+} \rightarrow$ $\Sigma^{+}$is topologically mixing. However, the above decomposition of $\Sigma^{+}=$ $\Sigma_{A_{0}}^{+} \cup \cdots \cup \Sigma_{A_{p-1}}^{+}$, with $\sigma^{p}$ topologically mixing on each component, together with the regularity of the function $f$, shows that the same definition may be made in the topologically transitive case. 
It is immediate from the definition that if $f \leq f^{\prime}$ then $P_{\mathrm{Gur}}(f, \sigma) \leq$ $P_{\text {Gur }}\left(f^{\prime}, \sigma\right)$ and that for any constant $c \in \mathbb{R}$ we have $P_{\text {Gur }}(f+c, \sigma) \leq$ $P_{\text {Gur }}(f, \sigma)+c$. We say that $f$ and $f^{\prime}$ are cohomologous if their difference takes the form $f-f^{\prime}=u \circ \sigma-u$. It is also clear from the definition that if $f$ and $f^{\prime}$ are cohomologous then $P_{\mathrm{Gur}}(f, \sigma)=P_{\mathrm{Gur}}\left(f^{\prime}, \sigma\right)$. We note the following useful lemma.

Lemma 3.2 If $r: \Sigma^{+} \rightarrow \mathbb{R}$ and $f: \Sigma^{+} \rightarrow \mathbb{R}$ are locally Hölder and $r$ is cohomolgous to a function $r^{\prime}$ satisfying $\inf _{x \in \Sigma^{+}} r^{\prime}(x)>0$ then $s \mapsto$ $P_{\mathrm{Gur}}(-s r+f, \sigma)$ is strictly decreasing.

Proof If $r$ and $r^{\prime}$ are cohomologous then, for any $s \in \mathbb{R},-s r$ and $-s r^{\prime}$ are cohomologous. Let $c=\inf _{x \in \Sigma^{+}} r^{\prime}(x)>0$ and suppose $t>s$. Then

$$
-t r^{\prime}=-s r-(t-s) r^{\prime} \leq-s r-(t-s) c
$$

and therefore

$$
\begin{aligned}
P_{\mathrm{Gur}}(-t r+f, \sigma) & =P_{\mathrm{Gur}}\left(-t r^{\prime}+f, \sigma\right) \\
& \leq P_{\mathrm{Gur}}\left(-s r^{\prime}-(t-s) c+f, \sigma\right) \\
& =P_{\mathrm{Gur}}\left(-s r^{\prime}+f, \sigma\right)-(t-s) c \\
& <P_{\mathrm{Gur}}\left(-s r^{\prime}+f, \sigma\right)=P_{\mathrm{Gur}}(-s r+f, \sigma) .
\end{aligned}
$$

We now specialise to the case where $S$ is finite. In this situation, we call $\sigma: \Sigma^{+} \rightarrow \Sigma^{+}$a (one-sided) subshift of finite type. The above definitions and results hold. If $f: \Sigma^{+} \rightarrow \mathbb{R}$ is Hölder continuous then $f$ is locally Hölder. Provided $\sigma: \Sigma^{+} \rightarrow \Sigma^{+}$is topologically transitive, the Gurevič pressure $P_{\text {Gur }}(f, \sigma)$ agrees with the standard pressure $P(f, \sigma)$, defined by

$$
P(f, \sigma)=\limsup _{n \rightarrow \infty} \frac{1}{n} \log \sum_{\sigma^{n} x=x} e^{f^{n}(x)}
$$

and if $\sigma$ is topologically mixing then the lim sup may be replaced with a limit.

We now consider skew product extensions of a shift of finite type $\sigma: \Sigma^{+} \rightarrow$ $\Sigma^{+}$, which we will assume to be topologically mixing. Let $G$ be a countable group (with identity element $e$ ) and let $\psi: \Sigma^{+} \rightarrow G$ be a function depending only on two co-ordinates, $\psi(x)=\psi\left(x_{0}, x_{1}\right)$.

(One could consider more general $\psi$ but this set-up suffices for our application to Anosov flows.) This data defines a group extension or skew product extension $T_{\psi}: \Sigma^{+} \times G \rightarrow \Sigma^{+} \times G$ by $T_{\psi}(x, g)=(\sigma x, g \psi(x))$. For $n \geq 1$ define $\psi_{n}$ by

$$
\psi^{n}(x)=\psi(x) \psi(\sigma x) \cdots \psi\left(\sigma^{n-1} x\right)
$$


then $T_{\psi}^{n}(x, g)=(x, g)$ if and only if $\sigma^{n} x=x$ and $\psi_{n}(x)=e$.

The map $T_{\psi}: \Sigma^{+} \times G \rightarrow \Sigma^{+} \times G$ is itself a countable state Markov shift with alphabet $S \times G$ and transition matrix $\widetilde{A}$ defined by $\widetilde{A}((i, g),(j, h))=1$ if $A(i, j)=1$ and $\psi(i, j)=g^{-1} h$, and $\widetilde{A}((i, g),(j, h))=0$ otherwise. Clearly, $\widetilde{A}$ is locally finite and so the topological transitivity and topological mixing of $\widetilde{\sigma}$ are equivalent to $\widetilde{A}$ being irreducible and aperiodic, respectively.

Let $f: \Sigma^{+} \rightarrow \mathbb{R}$ be Hölder continuous and define $\widetilde{f}: \Sigma_{A}^{+} \times G \rightarrow \mathbb{R}$ by $\tilde{f}(x, g)=f(x)$; then $\tilde{f}$ is locally Hölder continuous and its Gurevič pressure $P_{\text {Gur }}\left(\tilde{f}, T_{\psi}\right)$ is defined. In fact, it is easy to see that, due to the mixing of $\sigma$,

$$
P_{\mathrm{Gur}}\left(\widetilde{f}, T_{\psi}\right)=\limsup _{n \rightarrow \infty} \frac{1}{n} \log \sum_{\substack{\sigma^{n} x=x \\ \psi^{n}(x)=e}} e^{f^{n}(x)}
$$

We end this section by discussing two-sided subshifts of finite type and suspended flows over them. Given a finite alphabet $S$ and transition matrix $A$, we define

$$
\Sigma=\Sigma_{A}=\left\{x=\left(x_{n}\right)_{n=0}^{\infty} \in S^{\mathbb{Z}}: A\left(x_{n}, x_{n+1}\right)=1 \forall n \in \mathbb{Z}\right\}
$$

and the (two-sided) shift of finite type $\sigma: \Sigma \rightarrow \Sigma$ by $(\sigma x)_{n}=x_{n+1}$. As before, we give $\Sigma$ with the product topology induced by the discrete topology on $S$ and this is compatible with the metric $d(x, y)=2^{-n(x, y)}$, where

$$
n(x, y)=\inf \left\{|n|: x_{n} \neq y_{n}\right\}
$$

with $n(x, y)=\infty$ if $x=y$. Then $\Sigma$ is compact and $\sigma$ is a homeomorphism. There is an obvious one-to-one correspondence between the periodic points of $\sigma: \Sigma \rightarrow \Sigma$ and $\sigma: \Sigma^{+} \rightarrow \Sigma^{+}$. Furthermore, we may pass from Hölder continuous functions on $\Sigma$ to Hölder continuous functions on $\Sigma^{+}$in such a way that sums around periodic orbits are preserved. More precisely, we have the following lemma, due originally to Sinai [41], which appears as Proposition 1.2 of [28].

Lemma 3.3 Let $f: \Sigma \rightarrow \mathbb{R}$ be Hölder continuous. Then there is a Hölder continuous function $f^{\prime}: \Sigma^{+} \rightarrow \mathbb{R}$ (with a smaller Hölder exponent) such that $f^{n}(x)=\left(f^{\prime}\right)^{n}(x)$, whenever $\sigma^{n} x=x$.

We may also define suspended flows over $\sigma: \Sigma_{A} \rightarrow \Sigma_{A}$. Given a strictly positive continuous function $r: \Sigma \rightarrow \mathbb{R}^{+}$, we define the $r$-suspension space

$$
\Sigma^{r}=\{(x, s): x \in \Sigma, 0 \leq s \leq r(x)\} / \sim,
$$


where $(x, r(x)) \sim(\sigma x, 0)$. The suspended flow $\sigma_{r}^{t}: \Sigma^{r} \rightarrow \Sigma^{r}$ is defined by $\sigma_{r}^{t}(x, s)=(x, s+t)$ modulo the identifications. Clearly, there is a natural one-to-one correspondence between periodic orbits for $\sigma_{r}^{t}: \Sigma^{r} \rightarrow \Sigma^{r}$ and periodic orbits for $\sigma: \Sigma \rightarrow \Sigma$.

Furthermore, if $\gamma$ is a periodic $\sigma_{r}$-orbit corresponding to the periodic $\sigma$-orbit $\left\{x, \sigma x, \ldots, \sigma^{n-1} x\right\}$ then the period of $\gamma$ is equal to $r^{n}(x)$.

\section{Gurevič entropy for group extensions}

In this section, we initiate the comparison between Gurevič entropy and Gurevič pressure for group extensions of subshifts of finite type, and entropy and pressure for the base transformation and the abelianized extention. Carefully combining the result of Stadlbauer [42] and the results of [32] will produce one direction of a proof of Theorem 1.3. The other direction will lead us to prove a new result on Gurevič pressure, which is appears in Sect. 5.

Let $\sigma: \Sigma^{+} \rightarrow \Sigma^{+}$be a one-sided subshift of finite type. For a countable group $G$ and a function $\psi: \Sigma \rightarrow G$, we consider the group extension

$$
T_{\psi}: \Sigma \times G \rightarrow \Sigma \times G:(x, g) \mapsto(\sigma x, g \psi(x)),
$$

which we assume to be transitive.

Let $f: \Sigma^{+} \rightarrow \mathbb{R}$ be a Hölder continuous function. We will also use $f$ to denote the function on $\Sigma^{+} \times G$ defined by $f(x, g)=f(x)$. We wish to study the asymptotics of periodic points for $T_{\psi}$ (and compare them with those for $\sigma$ ) when they are weighted by $f$. Of course (provided $G$ is infinite), $T_{\psi}$ will have infinitely many periodic points with the same period but we will restrict to periodic points for which the second co-ordinate in the identity element.

In other words, we wish to compare the Gurevič pressure $P_{\text {Gur }}\left(f, T_{\psi}\right)$ with the pressure $P(f, \sigma)$. It is clear that $P_{\text {Gur }}\left(f, T_{\psi}\right) \leq P(f, \sigma)$ and it is natural to ask when equality holds.

This question has received considerable attention when the system and function exhibit a natural "time-reversal" symmetry. Suppose there is a fixed point free involution $\kappa: S \rightarrow S$ such that $A(\kappa j, \kappa i)=A(i, j)$, for all $i, j \in S$. We say that the skew product $T_{\psi}: \Sigma^{+} \times G \rightarrow \Sigma^{+} \times G$ is symmetric (with respect to $\kappa)$ if $\psi(\kappa j, \kappa i)=\psi(i, j)^{-1}$. A function $f: \Sigma^{+} \rightarrow \mathbb{R}$ is called weakly symmetric if, for all $n \geq 1$ and and all length $n$ cylinders $\left[z_{0}, z_{1}, \ldots, z_{n-1}\right]$, there exists $D_{n}>0$ such that $\lim _{n \rightarrow \infty} D_{n}^{1 / n}=1$ and

$$
\sup _{\substack{x \in\left[z_{0}, \ldots, z_{n-1}\right] \\ y \in\left[\kappa z_{n-1}, \ldots, \kappa z_{0}\right]}} \exp \left(f^{n}(x)-f^{n}(y)\right) \leq D_{n}
$$


The following is the main result of Stadlbauer [42], restricted to the case where the base is a (finite state) subshift of finite type. We will use this in subsequent arguments. (Stadlbauer considers skew product expansions of countable state Markov shifts satisfying the big images and pre-images property.) We include his more general result on the spectral radius of the transfer operator (Theorem 5.4 [42], there it is stated for pressure), the definition of which follows beneath the proposition.

Proposition 4.1 (Stadlbauer [42], Theorem 5.4, and Theorems 4.1 and 5.6) Let $T_{\psi}: \Sigma^{+} \times G \rightarrow \Sigma^{+} \times G$ be a transitive skew-product extension of a mixing subshift of finite type $\sigma: \Sigma^{+} \rightarrow \Sigma^{+}$by a countable group $G$.

If $G$ is non-amenable, then $\log \operatorname{spr}_{\mathcal{H}}\left(\mathcal{L}_{f}\right)<P(f, \sigma)$ for $f: \Sigma_{A}^{+} \rightarrow \mathbb{R}$ Hölder continuous.

If, in addition, $T_{\psi}: \Sigma^{+} \times G \rightarrow \Sigma^{+} \times G$ is assumed to be symmetric and $f: \Sigma_{A}^{+} \rightarrow \mathbb{R}$ is a weakly symmetric Hölder continuous function, then we have $P_{\mathrm{Gur}}\left(f, T_{\psi}\right)=P(f, \sigma)$ if and only if $G$ is amenable.

Remark 4.2 (i) In [42], Stadlbauer considers skew products with $\psi$ depending on only one co-ordinate. However, replacing $S$ by $\mathcal{W}^{2}$, one can easily recover the above formulation.

(ii) Setting $f=0$ immediately gives Theorem 1.3 for a symmetric extension.

In the absence of this symmetry, the answer becomes less clear. In order to elaborate on this problem, we introduce the transfer operator $\mathcal{L}_{f}$, defined pointwise by

$$
\mathcal{L}_{f} v(x, g)=\sum_{\substack{y \in \Sigma: \\ \sigma y=x}} e^{f(y)} v(y, g \psi(x))
$$

for $v: \Sigma \times G \rightarrow \mathbb{C}$. In order to make use of the spectral properties of this operator, we restrict it to the Banach space $\mathcal{H}$ of continuous functions $v$ for which $g \mapsto\|v(\cdot, g)\|_{\infty}$ is in $\ell^{2}(G)$, with norm

$$
\|v\|_{\mathcal{H}}=\left(\sum_{g \in G}\|v(\cdot, g)\|_{\infty}^{2}\right)^{1 / 2} .
$$

If $\psi$ and $f$ satisfy the above symmetry conditions then $P_{\text {Gur }}\left(f, T_{\psi}\right)$ is equal to the logarithm of $\operatorname{spr}_{\mathcal{H}}\left(\mathcal{L}_{f}\right)$, the spectral radius of $\mathcal{L}_{f}: \mathcal{H} \rightarrow \mathcal{H}$ [42]. However, this equality does not hold in general and without symmetry we may have $P_{\text {Gur }}\left(f, T_{\psi}\right)<\log \operatorname{spr}_{\mathcal{H}}\left(\mathcal{L}_{f}\right)$. On the other hand, there is a result of Jaerisch [20] that $\log \operatorname{spr}_{\mathcal{H}}\left(\mathcal{L}_{f}\right)=P(f, \sigma)$ if and only if $G$ is amenable, and so it is clear that, when considering $P_{\mathrm{Gur}}\left(f, T_{\psi}\right)$, the pressure $P(f, \sigma)$ does not 
provide a useful comparison. We shall show that, in fact, the natural comparison is between $P_{\mathrm{Gur}}\left(f, T_{\psi}\right)$ and $P_{\mathrm{Gur}}\left(f, T_{\psi^{\mathrm{ab}}}\right)$, where $T_{\psi^{\mathrm{ab}}}: \Sigma \times G^{\mathrm{ab}} \rightarrow \Sigma \times G^{\mathrm{ab}}$ is the induced $G^{\mathrm{ab}}$-extension, where $G^{\mathrm{ab}}=G /[G, G]$ is the abelianization of $G$. More precisely, if $\pi: G \rightarrow G^{\mathrm{ab}}$ is the natural projection then $\psi^{\mathrm{ab}}=\pi \circ \psi$.

We now address the Proof of Theorem 1.3. One implication in the theorem is given by the next proposition. The other implication will follow from the more general result proved in the next section (Theorem 5.1).

Proposition 4.3 If $G$ is not amenable then $h_{\mathrm{Gur}}\left(T_{\psi}\right)<h_{\mathrm{Gur}}\left(T_{\psi^{\mathrm{ab}}}\right)$.

Proof We have $G^{\mathrm{ab}}=\mathbb{Z}^{a} \times G_{0}$, for some $a \geq 0$, where $G_{0}$ is a finite abelian group. First suppose that $a>0$. The system $T_{\psi^{\mathrm{ab}}}: \Sigma^{+} \times G^{\mathrm{ab}} \rightarrow \Sigma^{+} \times G^{\mathrm{ab}}$ induces a system on $\Sigma^{+} \times \mathbb{Z}^{a}$, which we will still denote by $T_{\psi \text { ab }}$ and which has the same Gurevič entropy. Following the analysis of [32], $h_{\mathrm{Gur}}\left(T_{\psi^{\mathrm{ab}}}\right)=$ $P\left(\left(\left\langle\xi, \psi^{\mathrm{ab}}\right\rangle, \sigma\right)\right.$, for some $\xi \in \mathbb{R}^{a}$. Since $G$ is not amenable, the first part of Proposition 4.1 tells us that

$$
\log \operatorname{spr}_{\mathcal{H}}\left(\mathcal{L}_{\left\langle\xi, \psi^{\mathrm{ab}\rangle}\right\rangle}\right)<P\left(\left\langle\xi, \psi^{\mathrm{ab}}\right\rangle, \sigma\right)=h_{\mathrm{Gur}}\left(T_{\psi^{\mathrm{ab}}}\right) .
$$

and that, for any Hölder continuous $\left.f: \Sigma^{+} \rightarrow \mathbb{R}, P_{\text {Gur }}\left(f, T_{\psi}\right)\right) \leq$ $\log \operatorname{spr}_{\mathcal{H}}\left(\mathcal{L}_{f}\right)$. Combining these statements gives that

$$
\left.P_{\mathrm{Gur}}\left(\left\langle\xi, \psi^{\mathrm{ab}}\right\rangle, T_{\psi}\right)\right)<h_{\mathrm{Gur}}\left(T_{\psi^{\mathrm{ab}}}\right) .
$$

However, since $\psi^{n}(x)=e$ implies that $\left(\psi^{\mathrm{ab}}\right)^{n}=0$,

$$
\begin{aligned}
\left.P_{\mathrm{Gur}}\left(\left\langle\xi, \psi^{\mathrm{ab}}\right\rangle, T_{\psi}\right)\right) & =\limsup _{n \rightarrow \infty} \frac{1}{n} \log \sum_{\substack{\sigma^{n} x=x \\
\psi^{n}(x)=e}} e^{\left\langle\xi,\left(\psi^{\mathrm{ab}}\right)^{n}(x)\right\rangle} \\
& =\limsup _{n \rightarrow \infty} \frac{1}{n} \log \#\left\{x \in \Sigma^{+}: \sigma^{n} x=x,\left(\psi^{\mathrm{ab}}\right)^{n}=0\right\} \\
& =h_{\mathrm{Gur}}\left(T_{\psi}\right),
\end{aligned}
$$

giving the required strict inequality.

Now suppose that $a=0$, so that $G^{\mathrm{ab}}$ is finite. Then $h_{\mathrm{Gur}}\left(T_{\psi^{\mathrm{ab}}}\right)=h(\sigma)$. As above, we have

$$
h_{\mathrm{Gur}}\left(T_{\psi}\right) \leq \log \operatorname{spr}_{\mathcal{H}}\left(\mathcal{L}_{0}\right)<P(0, \sigma)=h(\sigma) \text {, }
$$

completing the proof. 


\section{Gurevič pressure for amenable extensions}

The purpose of this section is to prove the following result. Setting $f=0$ will complete the Proof of Theorem 1.3. To simplify notation, for a function $f: \Sigma^{+} \rightarrow \mathbb{R}$, we will use $f$ to denote the induced functions on the group extensions $\Sigma^{+} \times G$ and $\Sigma^{+} \times G^{\text {ab }}$ (i.e. $f(x, g)=f(x)$ for all group elements $g)$.

Theorem 5.1 Assume that $T_{\psi}$ is transitive. Let $f: \Sigma^{+} \rightarrow \mathbb{R}$ be Hölder continuous. If $G$ is amenable then

$$
P_{\text {Gur }}\left(f, T_{\psi}\right)=P_{\text {Gur }}\left(f, T_{\psi^{\mathrm{ab}}}\right) .
$$

The proof is inspired by Roblin's proof in [35] that if $\Gamma$ is a convex cocompact group of isometries of a CAT $(-1)$ space and $\Gamma^{\prime}$ is a normal subgroup such that $\Gamma / \Gamma^{\prime}$ is amenable then the critical exponents of $\Gamma$ and $\Gamma^{\prime}$ are equal. We will make use of a family of $\sigma$-finite measures $v_{\eta, g}$, indexed by $\Sigma^{+} \times G$, introduced by Stadlbauer [43]. For $t>0$, write

$$
\mathcal{P}(t)=\sum_{n \in \mathbb{N}} t^{-n} b_{n} \sum_{\substack{y \in \Sigma: \\ \sigma^{n} y=o}} e^{f^{n}(y)} \mathbb{1}_{\Sigma^{+} \times\{e\}}\left(T_{\psi}^{n}((y, e))\right.
$$

for a chosen distinguished $o \in \Sigma^{+}$, and where $b_{n}$ is a slowly diverging sequence chosen so that $\mathcal{P}(t)$ diverges at its radius of convergence. More precisely, if the terms $b_{n}$ are omitted then it is clear that the resulting series converges for $t>e^{P_{\mathrm{Gur}}\left(f, T_{\psi}\right)}$ and diverges for $t<e^{P_{\mathrm{Gur}}\left(f, T_{\psi}\right)}$. It is then possible to choose a non-decreasing sequence $b_{n} \geq 1$ such that $\lim _{n \rightarrow \infty} b_{n} / b_{n+1}=1$, $\mathcal{P}(t)$ has radius of convergence $e^{P_{\mathrm{Gur}}\left(f, \bar{T}_{\psi}\right)}$ and diverges at $t=e^{P_{\mathrm{Gur}}\left(f, T_{\psi}\right)}$ (Lemma 3.1 of [12]). For the rest of the section, we shall write $\rho:=e^{P_{\text {Gur }}\left(f, T_{\psi}\right)}$.

For $t>\rho, \eta \in \Sigma$ and $g \in G$, define a measure $v_{\xi, g}^{t}$ on $\Sigma^{+} \times G$ by the formula

$$
v_{\eta, g}^{t}(v):=\int_{\Sigma^{+} \times G} v d v_{\eta, g}^{t}=\frac{1}{\mathcal{P}(t)} \sum_{n \in \mathbb{N}} t^{-n} b_{n} \sum_{z \in \Sigma^{+} \times G: T_{\psi}^{n}(z)=(\eta, g)} e^{f^{n}(z)} v(z),
$$

for each continuous function $v: \Sigma^{+} \times G \rightarrow \mathbb{R}$. Now let $t \rightarrow \rho+$, and choose a weak limit $v_{\eta, g}$. We can do this because each $\Sigma^{+} \times\{g\}$ is compact, and $G$ is countable. We can also ensure that there is a countable dense subset of $\eta$ for which the limit is attained along the same subsequence. In Theorem 5.1 of [43], it is shown how to extend this to all $\eta \in \Sigma^{+}$using Hölder continuity. For the Proof of our Theorem 5.1, we will only use the countable collection of points 
$\eta=w o$, for $w \in \bigcup_{n \in \mathbb{N}} \mathcal{W}_{o}^{n}$, where $o$ is the chosen distinguished element of $\Sigma^{+}$and $\mathcal{W}_{o}^{n}$ denotes the set of elements $w$ of $\mathcal{W}^{n}$ for which $w o \in \Sigma^{+}$.

The following constants will frequently appear:

$$
B_{f}=\inf _{z \in \Sigma^{+}} e^{f(z)} \quad \text { and } \quad C_{f}=\exp \left(\frac{|f|_{\alpha}}{1-2^{-\alpha}}\right)
$$

where $\alpha>0$ is the Hölder exponent of $f$ and $|f|_{\alpha}$ is the $\alpha$-Hölder seminorm of $f$.

Lemma 5.2 (i) There exists $C>0$ such that, for any non-negative continuous function $v: \Sigma^{+} \times G \rightarrow \mathbb{R}$ and any $(\eta, g),(\xi, h) \in \Sigma^{+} \times G$ satisfying $T_{\psi}^{k}(\eta, g)=(\xi, h)$, we have

$$
v_{\eta, g}(v) \geq C^{k} v_{\xi, h}(v)
$$

(ii) For any non-negative continuous function $v: \Sigma^{+} \times G \rightarrow \mathbb{R}$ and any $\eta, \xi \in \Sigma^{+}$belonging to the same cylinder of length 1 , we have

$$
v_{\eta, g}(v) \geq C_{f}^{-1} v_{\xi, g}(v)
$$

Proof Let $v: \Sigma^{+} \times G \rightarrow \mathbb{R}$ be an indicator function on some cylinder. It will be sufficient to prove the lemma for functions of this form, the general nonnegative, continuous case follows by approximating by linear combinations of indicator functions.

We proceed with part (i). Let $(\eta, g),(\xi, h) \in \Sigma^{+} \times G$ with $T_{\psi}^{k}(\eta, g)=$ $(\xi, h)$. For $t>\rho$ we have,

$$
\begin{aligned}
v_{\eta, g}^{t}(v) & =\frac{1}{\mathcal{P}(t)} \sum_{n=1}^{\infty} t^{-n} b_{n} \sum_{w \in \mathcal{W}_{\eta}^{n}} e^{f^{n}(w \eta)} v\left(w \eta, g \psi^{n}(w)\right) \\
& \geq \frac{1}{\mathcal{P}(t)} \sum_{n=k+1}^{\infty} t^{-k} t^{-(n-k)} \frac{b_{n}}{b_{n-k}} b_{n-k} \sum_{u \in \mathcal{W}_{\xi}^{n-k}} e^{f^{k}(\xi)} e^{f^{n-k}(u \xi)} v\left(u \xi, h \psi^{n-k}(u)\right) \\
& \geq\left(\sup _{n \in \mathbb{N}} \frac{b_{n}}{b_{n-1}}\right)^{k} B_{f}^{k} t^{-k} \frac{1}{\mathcal{P}(t)} \sum_{m=1}^{\infty} t^{-m} b_{m} \sum_{u \in \mathcal{W}_{\xi}^{m}} e^{f^{m}(u \xi)} v\left(u \xi, h \psi^{m}(u)\right) .
\end{aligned}
$$

Taking weak limits as $t \rightarrow \rho+$ gives the conclusion (with $C=B_{f}\left(\sup _{n \in \mathbb{N}}\right.$ $\left.b_{n} / b_{n-1}\right)$ ).

Now, for part (ii) we assume that $\eta, \xi$ belong to the same cylinder. Recall that $v$ is assumed to be an indicator function, we write $v=\mathbb{1}_{[u]}$ for some $u$, and 
let $k$ be the length of $u$. For $t>\rho$ we have,

$$
\begin{aligned}
v_{\eta, g}^{t}(v)= & \frac{1}{\mathcal{P}(t)} \sum_{n=1}^{\infty} t^{-n} b_{n} \sum_{w \in \mathcal{W}_{\eta}^{n}} e^{f^{n}(w \eta)} v\left(w \eta, g \psi^{n}(w)\right) \\
= & \frac{1}{\mathcal{P}(t)} \sum_{n=1}^{\infty} t^{-n} b_{n} \sum_{w \in \mathcal{W}_{\xi}^{n}} e^{f^{n}(w \eta)-f^{n}(w \xi)} e^{f^{n}(w \xi)} v\left(w \eta, g \psi^{n}(w)\right) \\
\geq & \frac{1}{\mathcal{P}(t)} \sum_{n=k}^{\infty} t^{-n} b_{n} \sum_{w \in \mathcal{W}_{\xi}^{n}} C_{f}^{-1} e^{f^{n}(w \xi)} v\left(w \xi, g \psi^{n}(w)\right) \\
= & C_{f}^{-1} \frac{1}{\mathcal{P}(t)} \sum_{m=1}^{\infty} t^{-m} b_{m} \sum_{w \in \mathcal{W}_{\xi}^{m}} e^{f^{m}(w \xi)} v\left(w \xi, g \psi^{m}(w)\right) \\
& -\frac{1}{\mathcal{P}(t)} \sum_{i=1}^{k} t^{-i} b_{i} \sum_{w \in \mathcal{W}_{\xi}^{i}} C_{f}^{-1} e^{f^{i}(w \xi)} v\left(w \xi, g \psi^{i}(w)\right)
\end{aligned}
$$

Since $\mathcal{P}(t) \rightarrow \infty$, as $t \rightarrow \rho+$, it follows that,

$$
v_{\eta, g}^{t}(v) \geq C_{f}^{-1} v_{\xi, g}^{t}(v)
$$

as required.

Lemma 5.3 Let $v$ be a non-negative continuous function which is strictly positive on $\Sigma^{+} \times\{e\}$. If $T_{\psi}$ is transitive then $v_{z, g}(v)>0$. Furthermore, for each $a \in G$, we have

$$
\sup _{g \in G} \frac{v_{z, g a}(v)}{v_{z, g}(v)}<\infty
$$

Proof Let $g, a \in G$ be arbitrary. We write $z_{0}$ for the first letter of $z \in \Sigma^{+}$.

If $T_{\psi}$ is transitive then there are $\eta \in\left[z_{0}\right], \xi \in[o]$ and $k_{1}, k_{2} \geq 0$ such that $T_{\psi}^{k_{1}}(\eta, g)=(z, g a)$ and $T_{\psi}^{k_{2}}(z, g a)=(\xi, e)$. Then, by Lemma 5.2, we have that

$$
v_{\eta, g}(v) \geq C^{k_{1}} v_{z, g a}(v) \geq C^{k_{1}} C^{k_{2}} v_{\xi, e}(v) .
$$

Since $v$ is bounded from below away from zero on $\Sigma^{+} \times\{e\}$, there is $c>0$ with $v_{\xi, e}(v) \geq c v_{\xi, e}\left(\Sigma^{+} \times\{e\}\right)$. Then, $v_{\xi, e}\left(\Sigma^{+} \times\{e\}\right) \geq C_{f}^{-1} v_{o, e}\left(\Sigma^{+} \times\{e\}\right)$, and 
by construction $v_{o, e}\left(\Sigma^{+} \times\{e\}\right)=1$. This gives the conclusion that $v_{z, g}(v)>0$ for all $g \in G$.

To obtain the final statement, we note that

$$
\frac{v_{z, g a}(v)}{v_{\eta, g}(v)} \leq C^{-k_{1}}
$$

Moreover, since $\eta, z$ are in the same cylinder, the second part of Lemma 5.2 gives that

$$
\frac{v_{z, g a}(v)}{v_{z, g}(v)} \leq C^{-k_{1}} C_{f} .
$$

Lemma 5.4 For any continuous function $v: \Sigma^{+} \times G \rightarrow \mathbb{R}$ and any $g, a \in G$, we have that

$$
\rho v_{\eta, g}(v)=\sum_{u \in \mathcal{W}_{\eta}^{1}} e^{f(u \eta)} v_{u \eta, g \psi(u)}(v) .
$$

Proof For $t>\rho$, we have

$$
\begin{aligned}
v_{\eta, g}^{t}(v)= & \frac{1}{\mathcal{P}(t)} \sum_{n=1}^{\infty} t^{-n} b_{n} \sum_{w \in \mathcal{W}_{\eta}^{n}} e^{f^{n}(z)} v\left(w \eta, g \psi^{n}(w)\right) \\
= & \frac{1}{\mathcal{P}(t)} \sum_{u \in \mathcal{W}_{\eta}^{1}} \sum_{n=1}^{\infty} t^{-n} b_{n} \sum_{w \in \mathcal{W}_{u \eta}^{n-1}} e^{f^{n}(w u \eta)} v\left(w u \eta, g \psi(u) \psi^{n-1}(w)\right) \\
= & \frac{1}{\mathcal{P}(t)} \sum_{u \in \mathcal{W}_{\eta}^{1}} t^{-1} e^{f(u \eta)} \sum_{n=1}^{\infty} \frac{b_{n}}{b_{n-1}} t^{-(n-1)} b_{n-1} \\
& \times \sum_{w \in \mathcal{W}_{u \eta}^{n-1}} e^{f^{n-1}(w u \eta)} v\left(w u \eta, g \psi(u) \psi^{n-1}(w)\right) .
\end{aligned}
$$

Letting $t \rightarrow \rho+$, we obtain

$$
v_{\eta, g}(v)=\rho^{-1} \sum_{u \in \mathcal{W}_{\eta}^{1}} e^{f(u \xi)} v_{u \eta, g \psi(u)}(v),
$$

where we have used the divergence of $\mathcal{P}(t)$ as $t \rightarrow \rho+$ and that $\lim _{n \rightarrow \infty} b_{n} / b_{n-1}=1$. 
To complete the Proof of Theorem 5.1, we introduce the trick of Roblin when $G$ is amenable we can almost project $v$ to an eigenfunction for $\Sigma$.

Proof of Theorem 5.1 Let $\mathfrak{M}$ be the Banach mean for $G$. We fix a basepoint $o \in \Sigma$. Let $v: \Sigma^{+} \times G$ be a non-negative continuous function. Since $T_{\psi}$ is transitive, iterating Lemma 5.4 gives that, for some $C$ independent of $n$,

$$
\rho^{n} v_{o, g}(v)=\sum_{u \in \mathcal{W}_{o}^{n}} e^{f^{n}(u o)} v_{u o, g \psi^{n}(u)}(v) \geq C \sum_{u \in \mathcal{W}_{o}^{n}} e^{f^{n}(u o)} v_{o, g} \psi^{n}(u)(v)
$$

(where we use the Lemma 5.2 to compare $v_{u o, g \psi^{n}(u)}(v)$ with $v_{o, g} \psi^{n}(u)(v)$ ).

We cannot apply the mean directly in the above inequality as $v_{o, g} \psi^{n}(u)(v)$ may not be bounded in $g$. Instead, Lemma 5.3 tells us that we may normalise by $v_{o, g}(v)$ to obtain a bounded function on $G$. Recall Jensen's inequality: let $(X, \mu)$ be a measure space and $h \in L^{1}(\mu)$. If $\phi: \mathbb{R} \rightarrow \mathbb{R}$ is convex, then

$$
\phi\left(\int h d \mu\right) \leq \int \phi \circ h d \mu
$$

The inequality is also true for the Banach mean $\mathfrak{M}$ in place of the countably additive $\int \cdot d \mu$, since the proof only uses monotonicity of the integral and linearity. The reverse inequality is given when $\phi$ is concave. We apply this with concave function $\phi=\log$ to obtain

$$
\begin{aligned}
C^{-1} \rho^{n} \mathfrak{M}\left[g \mapsto \frac{v_{o, g}(v)}{v_{o, g}(v)}\right] & \geq \mathfrak{M}\left[g \mapsto \sum_{u \in \mathcal{W}_{o}^{n}} e^{f^{n}(u o)} \frac{v_{o, g} \psi^{n}(u)}{v_{o, g}(v)}\right] \\
& =\sum_{u \in \mathcal{W}_{o}^{n}} e^{f^{n}(u o)} \mathfrak{M}\left[g \mapsto \frac{v_{o, g} \psi^{n}(u)(v)}{v_{o, g}(v)}\right] \\
& \geq \sum_{u \in \mathcal{W}_{o}^{n}} e^{f^{n}(u o)} \exp \mathfrak{M}\left[g \mapsto \log \frac{v_{o, g} \psi^{n}(u)}{v_{o, g}(v)}\right] .
\end{aligned}
$$

The last function is important to us and we give it a name: we define $\chi: G \rightarrow \mathbb{R}$ by

$$
\chi(a)=\mathfrak{M}\left[g \mapsto \log \frac{v_{o, g a}(v)}{v_{o, g}(v)}\right]
$$


We claim that $\chi$ is a homomorphism. To see this, we first check that $\chi(a b)=$ $\chi(a)+\chi(b)$. Firstly,

$$
\frac{v_{o, g b a}(v)}{v_{o, g}(v)}=\frac{v_{o, g b a}(v)}{v_{o, g b}(v)} \frac{v_{o, g b}(v)}{v_{o, g}(v)},
$$

and when we take the mean, by right invariance, we have

$$
\mathfrak{M}\left[g \mapsto \log \frac{v_{o, g b a}(v)}{v_{o, g b}(v)}\right]=\mathfrak{M}\left[g \mapsto \log \frac{v_{o, g a}(v)}{v_{o, g}(v)}\right]
$$

We also have to check that $\chi(e)=0$, but this is immediate.

We can now bound the Gurevič pressure. Since any homomorphism factors through the abelianisation of $G$, if $(x, 0) \in \Sigma^{+} \times G^{\mathrm{ab}}$ satisfies $T_{\psi^{\mathrm{ab}}}^{n}(x, 0)=$ $(x, 0)$ then $\chi\left(\psi^{n}(x)\right)=0$. For $u \in \mathcal{W}^{n}$, we write $u^{\infty}$ for the infinite concatenation of copies of $u$. Since the transition matrix $A$ is aperiodic, there exists an $N \geq 1$ such that, for every $n \geq 1$ and every $u \in \mathcal{W}^{n}$ such that $u^{\infty} \in \Sigma^{+}$, there exists an admissible word $u^{\#}$ of length $N+1$ such that $\left(u^{\#}\right)_{0}=u_{0}$ and $u u^{\#} \in \mathcal{W}_{o}^{n+N+1}$. Since $u^{\infty}$ and $u u^{\#} o$ agree in the first $n+1$ places, we have $\psi^{n}\left(u^{\infty}\right)=\psi^{n}\left(u u^{\#} o\right)$ and

$$
e^{f^{n}\left(u^{\infty}\right)} \leq C_{f} e^{f^{n}\left(u u^{\#} o\right)}
$$

Thus we have

$$
\begin{aligned}
\sum_{\substack{x \in \Sigma^{+}: \sigma^{n} x=x \\
T_{\psi^{n} \mathrm{ab}}(x, 0)=(x, 0)}} e^{f^{n}(x)} & =\sum_{\substack{\left.x \in \Sigma^{+}: \sigma^{n} x=x \\
T^{n}(x)=(x)=0\right)}} e^{\chi\left(\psi^{n}(x)\right)} e^{f^{n}(x)} \\
& =\sum_{\substack{u \in \mathcal{W}^{n}: u^{\infty} \in \Sigma^{+} \\
\left(\psi^{\mathrm{ab}}\right)^{n}\left(u^{\infty}\right)=0}} e^{\chi\left(\psi^{n}\left(u^{\infty}\right)\right)} e^{f^{n}\left(u^{\infty}\right)} \\
& \leq C_{f} \sum_{\substack{u \in \mathcal{W}^{n}: u^{\infty} \in \Sigma^{+} \\
\left(\psi^{\mathrm{ab}}\right)^{n}\left(u^{\infty}\right)=0}} e^{\left.\chi\left(\psi^{n}\left(u u^{\#} o\right)\right)\right)} e^{f^{n}\left(u u^{\#} o\right)} \\
& \leq C_{f} C_{0}^{-(N+1)} D^{-(N+1)} \sum_{w \in \mathcal{W}_{o}^{n+N+1}} e^{\chi\left(\psi^{n+N+1}(w o)\right)} e^{f^{n+N+1}(w o)} \\
& \leq C^{-1} C_{f} C_{0}^{-(N+1)} D^{-(N+1)} \rho^{n+N+1},
\end{aligned}
$$

where $D=\inf _{x \in \Sigma^{+}} e^{\chi(\psi(x))}$. We conclude that $e^{P_{\mathrm{Gur}}\left(f, T_{\psi^{\mathrm{ab}}}\right)} \leq \rho=$ $e^{P_{\mathrm{Gur}}\left(f, T_{\psi}\right)}$. The other inequality is trivially true, therefore $P_{\mathrm{Gur}}\left(f, T_{\psi}\right.$ ab $)=$ $P_{\text {Gur }}\left(f, T_{\psi}\right)$. 


\section{Symbolic dynamics for Anosov flows}

We begin by discussing the symbolic coding of Anosov flows (and, more generally, hyperbolic flows) introduced by Ratner [34] and Bowen [5]. Let $M$ be a smooth compact Riemannian manifold and let $\phi^{t}: M \rightarrow M$ be a $C^{1}$ flow. A closed, $\phi^{t}$-invariant set $\Lambda \subset M$ is said to be hyperbolic if there is a continuous, $D \phi^{t}$-invariant splitting of the tangent bundle

$$
T_{\Lambda}(M)=E^{0} \oplus E^{s} \oplus E^{u}
$$

and constants $C, \lambda>0$ such that $E^{0}$ the line bundle tangent to the flow direction and

(1) $\left\|D \phi^{t} v\right\| \leq C e^{-\lambda t}\|v\|$ for all $v \in E^{s}$;

(2) $\left\|D \phi^{-t} v\right\| \leq C e^{-\lambda t}\|v\|$ for all $v \in E^{u}$.

(We remark that this definition is independent of the choice of metric when $\Lambda$ is compact.) If $M$ is a hyperbolic set then we call $\phi^{t}: M \rightarrow M$ an Anosov flow. There exist examples of Anosov flows which are not transitive $[3,18]$ but we shall always assume that transitivity holds, so we have that $M=\Omega(\phi)$, the non-wandering set for $\phi$. By a fundamental result of Anosov [2], the set of periodic orbits of $\phi$ is dense in $\Omega(\phi)$ and hence, in our setting, in $M$.

We now describe some of the constructions which play an important role in the symbolic coding of transitive Anosov flows. For $x \in M$ define the (strong) local stable manifold $W_{\epsilon}^{s}(x)$ and (strong) local unstable manifold $W_{\epsilon}^{u}(x)$ by

$$
\begin{aligned}
& W_{\epsilon}^{s}(x)=\left\{y \in M: d\left(\phi^{t}(x), \phi^{t}(y)\right) \leq \epsilon \text { for all } t, \lim _{t \rightarrow \infty} d\left(\phi^{t}(x), \phi^{t}(y)\right)=0\right\}, \\
& W_{\epsilon}^{u}(x)=\left\{y \in M: d\left(\phi^{-t}(x), \phi^{-t}(y)\right) \leq \epsilon \text { for all } t, \lim _{t \rightarrow \infty} d\left(\phi^{-t}(x), \phi^{-t}(y)\right)=0\right\} .
\end{aligned}
$$

For small enough $\epsilon>0$, these sets are diffeomorphic to embedded disks of dimension $d^{s}$ and $d^{u}$, respectively, where $d^{s}+d^{u}=\operatorname{dim} M-1$. These sets give us a local product structure $[\cdot, \cdot]$. For sufficiently close $x, y$, we have that $W_{\epsilon}^{s}(x) \cap W_{\epsilon}^{u}\left(\phi^{t}(y)\right) \neq \varnothing$ for a unique $t \in[-\epsilon, \epsilon]$, and we define $[x, y]$ to be this intersection point.

Suppose that $D_{1}, \ldots, D_{k}$ are codimension 1 disks that form a local crosssection to the flow. We say that $R_{i} \subset \operatorname{int}\left(D_{i}\right)$ is a rectangle if $x, y \in R_{i}$ implies that $[x, y]=\phi^{t} z$, for some $z \in D_{i}, t \in[-\epsilon, \epsilon]$, where the interior is taken relative to $D_{i}$. We say that $R_{i}$ is proper if $\overline{\operatorname{int}\left(R_{i}\right)}=R_{i}$, where again the interior is taken relative to $D_{i}$.

Write $P$ for the Poincaré map $P: \bigcup_{i=1}^{k} R_{i} \rightarrow \bigcup_{i=1}^{k} R_{i}$. Write $W_{\epsilon}^{s}\left(x, R_{i}\right)$ and $W_{\epsilon}^{u}\left(x, R_{i}\right)$ for the projection of $W_{\epsilon}^{s}(x)$ and $W_{\epsilon}^{u}(x)$ onto $R_{i}$ respectively. We say that $\mathcal{R}=\left\{R_{1}, \ldots, R_{k}\right\}$ is a Markov section if 
(1) $x \in \operatorname{int}\left(R_{i}\right)$ and $P x \in \operatorname{int}\left(R_{j}\right)$ implies that $P\left(W_{\epsilon}^{s}\left(x, R_{i}\right)\right) \subset$ $\left.W_{\epsilon}^{s}\left(P x, R_{j}\right)\right)$; and

(2) $x \in \operatorname{int}\left(R_{i}\right)$ and $P^{-1} x \in \operatorname{int}\left(R_{j}\right)$ implies that $P^{-1}\left(W_{\epsilon}^{u}\left(x, R_{i}\right)\right) \subset$ $\left.W_{\epsilon}^{s}\left(P^{-1} x, R_{j}\right)\right)$.

Proposition 6.1 (Bowen [5], Ratner [34]) For all sufficiently small $\epsilon>0$, $\phi^{t}$ has a Markov section $\mathcal{R}=\left\{R_{1}, \ldots, R_{k}\right\}$ such that $\operatorname{diam}\left(R_{i}\right) \leq \epsilon$ for each $i$, and $\bigcup_{t \in[-\epsilon, \epsilon]} \phi^{t}\left(\cup_{i=1}^{k} R_{i}\right)=M$.

These Markov sections provide us with a "symbolic coding" for the geodesic flow. In the following, the Markov section $\mathcal{R}=\left\{R_{1}, \ldots, R_{k}\right\}$ plays the role of an alphabet for a subshift of finite type $\Sigma$ with transition matrix $A$, defined by $A(i, j)=1$ if there is $x \in \operatorname{int}\left(R_{i}\right)$ with $P x \in \operatorname{int}\left(R_{j}\right)$.

Proposition 6.2 (Bowen [5], Bowen and Ruelle [6]) There is a mixing subshift of finite type $\sigma: \Sigma \rightarrow \Sigma$ and a strictly positive Hölder continuous potential $r: \Sigma \rightarrow \mathbb{R}^{+}$such that the suspended flow $\sigma_{r}^{t}: \Sigma^{r} \rightarrow \Sigma^{r}$ is semi-conjugate to $\phi^{t}: M \rightarrow M$. More precisely, there exists a bounded-to-one surjective Hölder continuous function $\theta: \Sigma^{r} \rightarrow M$ such that $\theta \circ \sigma_{r}^{t}=\phi^{t} \circ \theta$. Furthermore, if $f: M \rightarrow \mathbb{R}$ is Hölder continuous then $f \circ \theta: \Sigma^{r} \rightarrow \mathbb{R}$ is Hölder continuous and $\theta$ is a measure theoretic isomorphism between the equilibrium states of $f \circ \theta$ and $f$. In particular, $P(f \circ \theta)=P(f)$.

The semi-conjugacy $\theta: \Sigma^{r} \rightarrow M$ is not in general a bijection, so results on counting orbits do not immediately translate between settings. In particular, there is overcounting of the periodic orbits that pass through the boundaries of the sections. However, this discrepancy may be accounted for by the following result of Bowen (extending work of Manning [27] in the diffeomorphism case).

Lemma 6.3 (Bowen [5]) There are finitely many additional subshifts of finite type $\sigma_{i}: \Sigma_{i} \rightarrow \Sigma_{i}, i=1, \ldots, q$, with corresponding strictly positive Hölder continuous functions $r_{i}: \Sigma_{i} \rightarrow \mathbb{R}$ and Hölder continuous maps $\theta_{i}: \Sigma_{i}^{r_{i}} \rightarrow$ $M$, which are bounded-to-one but not surjective, such that $\theta_{i} \circ \sigma_{r}^{t}=\phi^{t} \circ \theta_{i}$, $i=1, \ldots, q$, and such that, if $v(\phi, T)$ (respectively, $v\left(\sigma_{r}, T\right), v\left(\sigma_{r_{i}}, T\right)$ ) denotes the number of periodic $\phi$-orbits (respectively, $\sigma_{r}$-orbits, $\sigma_{r_{i}}$-orbits) with period equal to $T$ then

$$
v(\phi, T)=v\left(\sigma_{r}, T\right)+\sum_{i=1}^{q} \epsilon_{i} v\left(\sigma_{r_{i}}, T\right),
$$

with $\epsilon_{i} \in\{-1,1\}$.

Since the $\theta_{i}$ are not surjective, for a Hölder continuous function $F: M \rightarrow \mathbb{R}$ we have $P\left(F \circ \theta_{i}, \sigma_{r_{i}}\right)<P(F, \phi)$. (To see this we follow the argument in 
section 7.23 of [36]. First note that $\theta_{i}\left(\Sigma_{i}^{r_{i}}\right)$ is a closed $\phi$-invariant proper subset of $M$. Since $\theta_{i}$ is bounded-to-one, $P\left(F \circ \theta_{i}, \sigma_{r_{i}}\right) \leq P\left(\left.F\right|_{\theta_{i}\left(\Sigma_{i}^{r_{i}}\right)}, \phi\right)$. But since the equilibrium state of $F$ is fully supported, it is easy to see by the variational principle that $P\left(\left.F\right|_{\theta_{i}\left(\Sigma_{i}^{r_{i}}\right)}, \phi\right)<P(F, \phi)$.) In particular, the suspended flows $\sigma_{r_{i}}^{t}$ have topological entropy strictly less than $h$.

For $F: M \rightarrow \mathbb{R}$, write

$$
N_{\phi}(T, F)=\sum_{\substack{\gamma \in \mathcal{P}(\phi): \\ l(\gamma) \leq T}} \exp \left(\int_{\gamma} F\right)
$$

(with similar definitions for the other flows). Then we have the following corollary.

Corollary 6.4 For every Hölder continuous function $F: M \rightarrow \mathbb{R}$, we have

$$
N_{\phi}(T, F)=N_{\sigma_{r}}(T, F \circ \theta)+O\left(e^{h_{F}^{\prime} T}\right),
$$

for some $h_{F}^{\prime}<P(F, \phi)$. In particular,

$$
N_{\phi}(T,\langle\xi, \Psi\rangle)=N_{\sigma_{r}}(T,\langle\xi, \Psi \circ \theta\rangle)+O\left(e^{h^{\prime} T}\right),
$$

for some $h^{\prime}<P(\langle\xi, \Psi\rangle, \phi)$.

\section{Covers and group extensions}

The aim of this section is to interpret the quantities $h, h(X)$ and $h\left(X^{\mathrm{ab}}\right)$ in terms of a subshift of finite type and its group extensions.

Consider a regular cover $p_{X}: X \rightarrow M$ of $M$, with covering group $G$ (acting as isometries on $X$ ), and the lifted flow $\phi_{X}^{t}: X \rightarrow X$. We will now describe the procedure to "lift" the symbolic dynamics for $\phi^{t}: M \rightarrow M$ to $\phi_{X}^{t}: X \rightarrow X$. We use the decoration $\mathcal{R}^{M}=\left\{R_{1}^{M}, \ldots, R_{k}^{M}\right\}$ for the Markov section for $\phi^{t}: M \rightarrow M$. For every pair $(i, j)$ such that $A(i, j)=1$, there is a minimum $t_{i j}>0$ with $\phi^{t_{i j}}\left(R_{i}^{M}\right) \cap R_{j}^{M} \neq \varnothing$. These $t_{i j}$ are clearly independent of the covering $X$ and $\mathcal{R}^{M}$ may be chosen so that $t_{i j}$ is arbitrarily small.

We assume that the rectangles in $\mathcal{R}^{M}$ have sufficiently small diameter so that they are each contained in an open ball that is simply connected. For each $R^{M} \in \mathcal{R}^{M}$, fix a lift $R^{X} \subseteq X$. (Or more precisely, fix a connected open set $U^{M}$ containing $R^{M}$, and a connected lift $U^{X}$. Then $R^{X}$ is chosen to lie inside $U^{X}$.) Having chosen the flow time between rectangles, and diameter of rectangles to be sufficiently small, we deduce that there is a unique $g=g(i, j) \in G$ 
with $\phi^{t_{i j}}\left(R_{i}^{X}\right) \cap g R_{j}^{X} \neq \varnothing$. We now define a function $\psi: \Sigma \rightarrow G$ by $\psi(x)=g\left(x_{0}, x_{1}\right)$.

We will use the function $\psi$ to identify periodic orbits in $M$ that lift to periodic orbits in $X$. We precede this discussion by elaborating on the $G$ class of a closed curve. Let $x \in M$ be fixed. Given that the covering map $p: X \rightarrow M$ is fixed, we have an isomorphism $G=\pi_{1}(M, x) / N$, where $N$ is a normal subgroup of $\pi_{1}(M, x)$ (isomorphic to $\pi_{1}(X)$ ).

For an arbitrary closed curve $\gamma:[0, R] \rightarrow M$, we can choose paths connecting $x$ to $\gamma(0)$ to produce a closed curve based at $x$ and hence a homotopy class in $\pi_{1}(M, x)$. A different choice of path from $x$ to $\gamma(0)$ gives a conjugate element of $\pi_{1}(M, x)$. Thus we obtain a well-defined conjugacy class in $G$, which we will denote by $\langle\gamma\rangle_{X}$ and call the $G$-class of $\gamma$. We say that the $G$ class is trivial if $\langle\gamma\rangle_{X}$ consists only of the identity, in which case we slightly abuse notation by writing $\langle\gamma\rangle_{X}=e$, and this is equivalent to $\gamma$ lifting to a closed curve in $X$ Clearly, a closed curve $\gamma$ has trivial $G$-class if any choice of path $x$ to $\gamma(0)$ gives a closed curve with homotopy class in $N$.

We claim that $\psi$ codes orbits with trivial $G$-class, but we can only make statements about points for which the semi-conjugacy $\theta$ is actually a bijection. Write $\mathcal{P}(\phi)^{*}$ for the set of periodic orbits in $M$ for which $\theta$ induces a bijection.

Proposition 7.1 Suppose that $\left\{x, \sigma x, \ldots, \sigma^{n-1} x\right\}$ is a periodic $\sigma$-orbit (with $\sigma^{n} x=x$ ) corresponding to $\gamma \in \mathcal{P}(\phi)^{*}$. Then $\gamma$ has trivial $G$-class if and only if $\psi^{n}(x)=e$.

Proof We can regard $\theta(x, 0)$ as the initial point on $\gamma$. Let $\tilde{\gamma}$ be a lift of $\gamma$, we may assume starts in $R_{x_{0}}^{X}$. By construction, $\tilde{\gamma}$ ends in $\psi^{n}(x)$ and is thus periodic if and only if $\psi^{n}(x)=e$.

Now suppose that $Y=X^{\mathrm{ab}}$ is the maximal abelian subcover of $X$, with abelian covering group $G^{\mathrm{ab}}=G /[G, G]$. Then $\langle\gamma\rangle_{Y}=[\gamma]$. We define $\psi_{Y}$ : $\Sigma \rightarrow G^{\mathrm{ab}}$ in an analogous way to $\psi: \Sigma \rightarrow G$, i.e. $\psi_{Y}(x)$ is given by $\phi^{t_{x_{0} x_{1}}}\left(R_{x_{0}}^{Y}\right) \cap \psi_{Y}(x) R_{x_{1}}^{Y} \neq \varnothing$, where $R_{i}^{Y}$ is the projection of $R_{i}^{X}$ to $Y$. Clearly, $\psi_{Y}$ is the composition of $\psi$ and the projection homomorphism from $G$ to $G^{\mathrm{ab}}$. We immediately have the following.

Proposition 7.2 Suppose that $\left\{x, \sigma x, \ldots, \sigma^{n-1} x\right\}$ is a periodic $\sigma$-orbit (with $\sigma^{n} x=x$ ) corresponding to $\gamma \in \mathcal{P}(\phi)^{*}$. Then $[\gamma]=\psi_{Y}^{n}(x)$ (and, in particular, $[\gamma]=0$ if and only if $\left.\psi_{Y}^{n}(x)=0\right)$.

Proof This follows from Proposition 7.1 and the fact that $G^{\mathrm{ab}}$ is abelian.

We now wish to make two reductions. First of all, if $G^{\mathrm{ab}} \cong \mathbb{Z}^{a} \times G_{0}$ with $G_{0}$ a non-trivial finite abelian group then $Y$ is a finite cover of a manifold $Y^{\prime}$, which is a $\mathbb{Z}^{a}$-cover of $M$. By Lemma 2.7, $h(Y)=h\left(Y^{\prime}\right)$ and so there is no loss 
of generality in replacing $Y$ with $Y^{\prime}$ is our analysis. Slightly abusing notation, we shall still use $Y$ to denote this $\mathbb{Z}^{a}$-cover of $M$. Then $\langle\gamma\rangle_{Y}=[\gamma]=\int_{\gamma} \Psi$ is an element of $\mathbb{Z}^{a}$.

Secondly, wish to switch from the two-sided subshift of finite type $\sigma: \Sigma \rightarrow$ $\Sigma$ to the corresponding one-sided subshift of finite type $\sigma: \Sigma^{+} \rightarrow \Sigma^{+}$. There is an obvious identification between the periodic orbits of the two systems. Since the functions $\psi$ and $\psi_{Y}$ depend on only two co-ordinates, we can equally well regard them as functions defined on the one-sided shift space $\Sigma^{+}$. Also, by Lemma 3.3, we may change $r$ by the addition of a coboundary to obtain a function defined on $\Sigma^{+}$. We will continue to denote this modified function by $r$ and note that its sums around periodic orbits are unchanged.

After these two reductions, we have extended systems $T_{\psi}: \Sigma^{+} \times G \rightarrow$ $\Sigma^{+} \times G$ and $T_{\psi_{Y}}: \Sigma \times \mathbb{Z}^{a} \rightarrow \Sigma^{+} \times \mathbb{Z}^{a}$.

We end the section with two results on transitivity.

Lemma 7.3 If $\phi_{X}^{t}: X \rightarrow X$ is transitive, then $T_{\psi}$ is transitive.

Proof Let $x \in X$ be a point with dense $\phi_{X}$-orbit. Without loss of generality $x \in \bigcup_{i=1}^{k} \bigcup_{g \in G} g \cdot R_{i}^{X}$ and then $\left\{P_{X}^{n} x\right\}_{n=-\infty}^{\infty}$ is dense in $\bigcup_{i=1}^{k} \bigcup_{g \in G} g$. $R_{i}^{X}$, where $P_{X}$ is the Poincaré map between the sections in $X$. Suppose that $\widetilde{A}\left(\left(i_{j}, g_{j}\right),\left(i_{j+1}, g_{j+1}\right)\right)=1$, where $\widetilde{A}$ is the transition matrix for $\Sigma^{+} \times G$, for $j=0, \ldots, n$. Then

$$
U=\bigcap_{j=0}^{n} P_{X}^{-j}\left(\operatorname{int}\left(g_{j} \cdot R_{i_{j}}^{X}\right)\right)
$$

is non-empty and open in $\bigcup_{i=1}^{k} \bigcup_{g \in G} g \cdot R_{i}^{X}$. (Here $\operatorname{int}\left(g_{j} \cdot R_{i_{j}}^{X}\right)$ is taken with respect to the co-dimension one disk containing $g_{j} \cdot R_{i_{j}}^{X}$.) Since $x$ has dense $P_{X}$-orbit, $P_{X}^{m} x \in U$ for some $m \in \mathbb{Z}$. Then $P_{X}^{m+j}(x) \in \operatorname{int}\left(g_{j} \cdot R_{i_{j}}^{X}\right)$ for $j=0, \ldots, n$. By definition, this implies that the $T_{\psi}$-orbit of $\left(\theta\left(p_{X}(x)\right), g_{0}\right) \in$ $\Sigma^{+} \times G\left(\right.$ where $\theta\left(p_{X}(x)\right)$ is identified with a point in the one-sided shift) passes through the (arbitrary) cylinder $\left[\left(i_{0}, g_{0}\right), \ldots,\left(i_{n}, g_{n}\right)\right]$ and is thus dense in $\Sigma^{+} \times G$. Therefore, $\widetilde{\sigma}: \Sigma^{+} \times G \rightarrow \Sigma^{+} \times G$ is transitive.

Lemma 7.4 If $\phi_{Y}^{t}: Y \rightarrow Y$ is transitive then the Anosov flow $\phi^{t}: M \rightarrow M$ is $Y$-full.

Proof Suppose that $\phi_{Y}$ is transitive. Then, by Lemma 7.3, $T_{\psi_{Y}}: \Sigma^{+} \times \mathbb{Z}^{a} \rightarrow$ $\Sigma^{+} \times \mathbb{Z}^{a}$, is transitive. In addition,

$$
\bigcup_{n=1}^{\infty}\left\{\psi_{Y}^{n}(x): \sigma^{n} x=x\right\}=\{[\gamma]: \gamma \in \mathcal{P}(\phi)\},
$$


Suppose $\phi$ is not $Y$-full. Then, by Proposition 2.4, $\bigcup_{n=1}^{\infty}\left\{\psi_{Y}^{n}(x): \sigma^{n} x=x\right\}$ is contained in a closed half-space in $\mathbb{R}^{a}$ and hence there exists $t \in \mathbb{R}^{a} \backslash\{0\}$ such that $\left\langle t, \psi_{Y}^{n}(x)\right\rangle \geq 0$, whenever $\sigma^{n} x=x$. Since measures supported on periodoc orbits are dense in the space of $\sigma$-invariant probability measaures, this gives that $\int\left\langle t, \psi_{Y}\right\rangle d m \geq 0$ for every $\sigma$-invariant probability measure $m$. By a result of Savchenko [38], this implies that there exists a continuous function $u: \Sigma \rightarrow \mathbb{R}$ such that $\left\langle t, \psi_{Y}\right\rangle+u \circ \sigma-u \geq 0$. Hence, for all $n \geq 1$, $\left\langle t, \psi_{Y}^{n}\right\rangle+u \circ \sigma^{n}-u \geq 0$, giving $\left\langle t, \psi_{Y}^{n}\right\rangle \geq-2\|u\|_{\infty}$. Since $T_{\psi_{Y}}^{n}(x, k)=$ $\left(\sigma^{n} x, k+\psi_{Y}^{n}(x)\right)$, if we choose $\alpha \in \mathrm{Z}^{a}$ with $\langle t, \alpha\rangle<-2\|u\|_{\infty}$, then no $T_{\psi_{Y}}$-orbit starting in $\Sigma \times\{0\}$ can reach $\Sigma \times\{\alpha\}$, and so $T_{\psi_{Y}}$ is not transitive.

\section{Proof of Theorem 1.1}

We begin with a lemma relating the quantities $h(X)$ and $h(Y)$ to Gurevič pressures for $T_{\psi}$ and $T_{\psi_{Y}}$. Let $\sigma: \Sigma^{+} \rightarrow \Sigma^{+}$be the one-sided subshift of finite type and let $r: \Sigma^{+} \rightarrow \mathbb{R}$ (cohomologous to a strictly positive function), $\psi=\psi_{X}: \Sigma^{+} \rightarrow G$ and $\psi_{Y}: \Sigma^{+} \rightarrow \mathbb{Z}^{a}$ be the functions constructed in the previous section.

We have the following key lemma.

Lemma 8.1 The following three statements hold:

(1) $h=P(0, \phi)$ is the unique zero of $s \mapsto P(-s r, \sigma)$;

(2) $h(Y)=P(\langle\xi, \Psi\rangle, \phi)$, and $h(Y)$ is the unique zero of $s \mapsto P_{\mathrm{Gur}}(-s r+$ $\left.\langle\xi, \Psi\rangle, T_{\psi_{Y}}\right)$

(3) $h(X)=h(Y)$ if and only if $P_{\mathrm{Gur}}\left(-h(Y) r+\left\langle\xi, \psi_{Y}\right\rangle, T_{\psi}\right)=0$.

Proof Part (1) is a standard result, see [28].

Let us now prove (2). By Lemma 2.7, we know that $h(Y)=P(\langle\xi, \Psi\rangle, \phi)$. Also, by Lemma 2.8, $h(Y)$ is the abscissa of convergence of

$$
\sum_{\substack{\gamma \in \mathcal{P}(\phi): \\[\gamma]=0}} \frac{\Lambda(\gamma)}{l(\gamma)} e^{-s l(\gamma)}=\sum_{\substack{\gamma \in \mathcal{P}(\phi): \\[\gamma]=0}} \frac{\Lambda(\gamma)}{l(\gamma)} e^{-s l(\gamma)+\langle\xi,[\gamma]\rangle}
$$

By Corollary 6.4,

$$
\sum_{\substack{\gamma \in \mathcal{P}(\phi): \\[\gamma]=0}} \frac{\Lambda(\gamma)}{l(\gamma)} e^{-s l(\gamma)+\langle\xi,[\gamma]\rangle}
$$


differs from

$$
\sum_{n=1}^{\infty} \frac{1}{n} \sum_{\substack{\sigma^{n} x=x: \\ \psi_{Y}^{n}(x)=0}} e^{-s r^{n}(x)}
$$

by a series with abscissa of convergence $h^{\prime}<P(\langle\xi, \Psi\rangle, \phi)=h(Y)$. Therefore $P_{\text {Gur }}\left(-h(Y) r+\langle\xi, \Psi\rangle, T_{\psi_{Y}}\right)=0$. Since $r$ is strictly positive, Lemma 3.2 gives that $s \mapsto P_{\mathrm{Gur}}\left(-s r, T_{\psi_{Y}}\right)$ is strictly decreasing and therefore $h(Y)$ is the unique zero.

Finally, we prove (3). Write

$$
S_{1}(s):=\sum_{\substack{\gamma \in \mathcal{P}(\phi): \\\langle\gamma\rangle=0}} e^{-s l(\gamma)} \text { and } S_{2}(s):=\sum_{n=1}^{\infty} \frac{1}{n} \sum_{\substack{\sigma^{n} x=x: \\ \psi^{n}(x)=e}} e^{-s r^{n}(x)}
$$

We have $h(Y)=h(X)$ if and only if $h(Y)$ is the abscissa of convergence of $S_{1}(s)$. Since $\langle\gamma\rangle=e$ implies that $[\gamma]=0$, we can use argument in the proof of Lemma 2.8 and Corollary 6.4 to show that the abscissa of convergence of $S_{1}(s)-S_{2}(s)$ is bounded above by $\max \left\{h(Y) / 2, h^{\prime}\right\}<h(Y)$. Hence, $S_{1}(s)$ has abscissa of convergence $h(Y)$ if and only if $S_{2}(s)$ has abscissa of convergence $h(Y)$, which in turn is equivalent to $P_{\mathrm{Gur}}\left(-h(Y) r, T_{\psi}\right)=0$. But, since $\psi^{n}(x)=e$ implies that $\psi_{Y}^{n}(x)=0$, we can employ the argument used to prove (2) to show that, for any $s \in \mathbb{R}$,

$$
P_{\mathrm{Gur}}\left(-s r+\left\langle\xi, \psi_{Y}\right\rangle, T_{\psi}\right)=P_{\mathrm{Gur}}\left(-s r, T_{\psi}\right)
$$

completing the proof.

We now complete the Proof of Theorem 1.1.

Proof of Theorem 1.1 We first deal with the case $a>0$. Suppose that $G$ is non-amenable. By Theorem 1.1 of [20], since $G$ is non-amenable, we have that

$$
\log \operatorname{spr}_{\mathcal{H}}\left(\mathcal{L}_{-h(Y) r+\left\langle\xi, \psi_{Y}\right\rangle}\right)<P\left(-h(Y) r+\left\langle\xi, \psi_{Y}\right\rangle, \sigma\right)
$$

Moreover, by the same theorem, for any Hölder continuous $f: \Sigma^{+} \rightarrow \mathbb{R}$, we always have $P_{\text {Gur }}\left(f, T_{\psi}\right) \leq \log \operatorname{spr}_{\mathcal{H}}\left(\mathcal{L}_{f}\right)$. Putting these together gives

$$
\begin{aligned}
P_{\mathrm{Gur}}\left(-h(Y) r+\left\langle\xi, \psi_{Y}\right\rangle, T_{\psi}\right) & <P\left(-h(Y) r+\left\langle\xi, \psi_{Y}\right\rangle, \sigma\right) \\
& =P\left(-h(Y) r+\left\langle\xi, \psi_{Y}\right\rangle, T_{\psi_{Y}}\right),
\end{aligned}
$$


where the last identity follows from Lemma 8.1. Now, by the proof of part (3) of Lemma 8.1, this becomes $P_{\mathrm{Gur}}\left(-h(Y) r, T_{\psi}\right)<0$ and therefore $h(X)<h(Y)$.

Now suppose that $G$ is amenable. By Theorem 5.1, $P_{\mathrm{Gur}}\left(-s r+\left\langle\xi, \psi^{\mathrm{ab}}\right\rangle, T_{\psi}\right)$ $=P_{\mathrm{Gur}}\left(-s r+\left\langle\xi, \psi^{\mathrm{ab}}\right\rangle, T_{\psi^{\mathrm{ab}}}\right)$ for any $s$. By, Lemma 8.1, this implies that $h(X)=h(Y)$.

Finally, we deal with the case where $a=0$, which requires a simple modification of the above arguments. In this case, $G^{\mathrm{ab}}$ is finite and so $h(Y)=h$. If $G$ is non-amenable then

$$
P_{\text {Gur }}\left(-h r, T_{\psi}\right) \leq \log \operatorname{spr}_{\mathcal{H}}\left(\mathcal{L}_{-h r}\right)<P(-h r, \sigma)=0
$$

so $h(X)<h$. If $G$ is amenable then Theorem 5.1 gives

$$
P_{\mathrm{Gur}}\left(-s r, T_{\psi}\right)=P_{\mathrm{Gur}}\left(-s r, T_{\psi^{\mathrm{ab}}}\right)=P(-s r, \sigma),
$$

so $h(X)=h$.

\section{Equidistribution}

In this final section, we consider the spatial distribution of $\phi$-periodic orbits with trivial $G$-class, in the case where $G$ is amenable. This generalises Theorem 2 of [39] which deals with $G$ abelian. Write

$$
\Xi(T, \epsilon)=\#\{\gamma \in \mathcal{P}(\phi): T<l(\gamma) \leq T+\epsilon,\langle\gamma\rangle=e\} .
$$

Our results will be based on the following definition.

Definition 9.1 We say the $\phi$-periodic orbits with trivial $G$-class are equidistributed with respect to a measure $\mu$ if, for every $\epsilon>0$ and continuous function $F: M \rightarrow \mathbb{R}$, we have

$$
\lim _{T \rightarrow \infty} \frac{1}{\Xi(T, \epsilon)} \sum_{\substack{\gamma \in \mathcal{P}(\phi): \\ T<l(\gamma) \leq T+\epsilon,\langle\gamma\rangle=e}} \frac{1}{l(\gamma)} \int_{\gamma} F=\int F d \mu .
$$

We have the following equidistribution result for Anosov flows.

Theorem 9.2 Let $\phi^{t}: M \rightarrow M$ be an Anosov flow and let $X$ be a regular cover of $M$ with amenable covering group $G$. Suppose that the lifted flow $\phi_{X}^{t}: X \rightarrow X$ is transitive. Then the $\phi$-periodic orbits with trivial $G$-class are equidistributed with respect to $\mu_{\langle\xi, \Psi\rangle}$, the equilibrium state for $\langle\xi, \Psi\rangle$.

Specialising to geodesic flows gives the following corollaries. 
Corollary 9.3 Let $\phi^{t}: S V \rightarrow S V$ be the geodesic flow on the unit-tangent bundle over a compact negatively curved manifold $V$ and let $X$ be a regular cover of $M$ with amenable covering group $G$. Then the $\phi$-periodic orbits with trivial $G$-class are equidistributed with respect to the measure of maximal entropy for $\phi$.

Proof Since $\phi$ is a geodesic flow, $\xi=0$. Also, since $G$ is amenable, $G$ is not equal to $\pi_{1}(M)$ and hence, by a result of Eberlein [15], the lifted flow $\phi_{X}^{t}: X \rightarrow X$ is transitive. Thus the corollary follows from Theorem 9.2.

Corollary 9.4 Let $V$ be a compact manifold with negative sectional curvatures and let $\widehat{V}$ be a regular cover with amenable covering group $G$. Then the closed geodesics on $V$ with trivial $G$-class are are equidistributed with respect to the projection to $V$ of the measure of maximal entropy for the geodesic flow on the unit-tangent bundle $S V$.

If $V$ has constant negative curvature then the measure of maximal entropy is equal to the volume measure and so we have the following.

Corollary 9.5 Let $V$ be a compact hyperbolic manifold and let $\widehat{V}$ be a regular cover with amenable covering group $G$. Then the closed geodesics on $V$ with trivial $G$-class are are equidistributed with respect to the volume measure on $V$.

Theorem 9.2 is a consequence of the following large deviations result. For $\gamma \in \mathcal{P}(\phi)$, let $\mu_{\gamma}$ denote the $\phi$-invariant probability measure defined by

$$
\int F d \mu_{\gamma}=\frac{1}{l(\gamma)} \int_{\gamma} F
$$

Theorem 9.6 Let $\phi^{t}: M \rightarrow M$ be an Anosov flow and let $X$ be a regular cover of $M$ with amenable covering group $G$. Suppose that the lifted flow $\phi_{X}^{t}: X \rightarrow X$ is transitive. Then, for any weak*-compact set $\mathcal{K} \subset \mathcal{M}(\phi)$ such that $\mu_{\langle\xi, \Psi\rangle} \notin \mathcal{K}$, we have

$\limsup _{T \rightarrow \infty} \frac{1}{T} \log \left(\frac{\#\left\{\gamma \in \mathcal{P}(\phi): T<l(\gamma) \leq T+\epsilon,\langle\gamma\rangle=e, \mu_{\gamma} \in \mathcal{K}\right\}}{\#\{\gamma \in \mathcal{P}(\phi): T<l(\gamma) \leq T+\epsilon,\langle\gamma\rangle=e\}}\right)<0$.

Proof To shorten some formulae, we will write

$$
Q(F):=P(\langle\xi, \Psi\rangle+F, \phi) .
$$

We begin by noting the following. By Theorem 1.1,

$$
\lim _{T \rightarrow \infty} \frac{1}{T} \log \#\{\gamma \in \mathcal{P}(\phi): T<l(\gamma) \leq T+\epsilon,\langle\gamma\rangle=e\}=Q(0)
$$


and, for any continuous function $F: M \rightarrow \mathbb{R}$,

$$
\begin{aligned}
\sum_{\substack{\gamma \in \mathcal{P}(\phi) \\
T<l(\gamma) \leq T+\epsilon,\langle\gamma\rangle=e}} \exp \left(\int_{\gamma} F\right) & =\sum_{\substack{\gamma \in \mathcal{P}(\phi) \\
T<l(\gamma) \leq T+\epsilon,\langle\gamma\rangle=e}} \exp \left(\int_{\gamma}\langle\xi, \Psi\rangle+F\right) \\
& \leq \sum_{\substack{\gamma \in \mathcal{P}(\phi) \\
T<l(\gamma) \leq T+\epsilon}} \exp \left(\int_{\gamma}\langle\xi, \Psi\rangle+F\right),
\end{aligned}
$$

giving

$$
\lim _{T \rightarrow \infty} \frac{1}{T} \log \sum_{\substack{\gamma \in \mathcal{P}(\phi) \\ T<l(\gamma) \leq T+\epsilon,\langle\gamma\rangle=e}} \exp \left(\int_{\gamma} F\right) \leq Q(F) .
$$

Now define

$$
\rho=\rho_{\mathcal{K}}:=\inf _{\nu \in \mathcal{K}} \sup _{F \in C(M, \mathbb{R})}\left(\int F d v-Q(F)\right) .
$$

Fix $\delta>0$. For every $v \in \mathcal{K}$, there exists $F \in C(M, \mathbb{R})$ such that

$$
\int F d v-Q(F)>\rho-\delta .
$$

Thus we have

$$
\mathcal{K} \subset \bigcup_{F \in C(M, \mathbb{R})}\left\{v \in \mathcal{M}(\phi): \int F d v-Q(F)>\rho-\delta\right\} .
$$

Since $\mathcal{K}$ is weak* ${ }^{*}$-compact, we can find a finite subcover

$$
\mathcal{K} \subset \bigcup_{i=1}^{k}\left\{v \in \mathcal{M}(\phi): \int F_{i} d v-Q(F)>\rho-\delta\right\}
$$

This gives us the inequality

$$
\begin{aligned}
& \#\left\{\gamma \in \mathcal{P}(\phi): T<l(\gamma) \leq T+\epsilon,\langle\gamma\rangle=e, \mu_{\gamma} \in \mathcal{K}\right\} \\
& \leq \sum_{i=1}^{k} \#\left\{\gamma \in \mathcal{P}(\phi): T<l(\gamma) \leq T+\epsilon,\langle\gamma\rangle=e, \int F_{i} d \mu_{\gamma}-Q(F)>\rho-\delta\right\} \\
& \leq \sum_{i=1}^{k} \sum_{\substack{\gamma \in \mathcal{P}(\phi) \\
T<l(\gamma) \leq T+\epsilon,\langle\gamma\rangle=e}} \exp \left(-l(\gamma)\left(Q\left(F_{i}\right)+(\rho-\delta)\right)+\int_{\gamma} F_{i}\right)
\end{aligned}
$$




$$
\leq \sum_{i=1}^{k} \max \left\{e^{-T\left(Q\left(F_{i}\right)+(\rho-\delta)\right)}, e^{-(T+\epsilon)\left(Q\left(F_{i}\right)+(\rho-\delta)\right)}\right\} \sum_{\substack{\gamma \in \mathcal{P}(\phi) \\ T<l(\gamma) \leq T+\epsilon,\langle\gamma\rangle=e}} \exp \left(\int_{\gamma} F_{i}\right)
$$

and hence, using (9.2) we obtain the growth rate estimate

$$
\limsup _{T \rightarrow \infty} \frac{1}{T} \log \#\left\{\gamma \in \mathcal{P}(\phi): T<l(\gamma) \leq T+\epsilon,\langle\gamma\rangle=e, \mu_{\gamma} \in \mathcal{K}\right\} \leq-\rho+\delta .
$$

Since $\delta>0$ is arbitrary, we combine this with (9.1) to deduce that

$$
\limsup _{T \rightarrow \infty} \frac{1}{T} \log \left(\frac{\#\left\{\gamma \in \mathcal{P}(\phi): T<l(\gamma) \leq T+\epsilon,\langle\gamma\rangle=e, \mu_{\gamma} \in \mathcal{K}\right\}}{\#\{\gamma \in \mathcal{P}(\phi): T<l(\gamma) \leq T+\epsilon,\langle\gamma\rangle=e\}}\right) \leq-\rho-Q(0) .
$$

To complete the proof, we will show that $\rho+Q(0)>0$. First note that if $v \neq \mu\langle\xi . \Psi\rangle$ then

$$
\begin{aligned}
& \sup _{F \in C(M, \mathbb{R})}\left(\int F d v-Q(F)+Q(0)\right) \\
= & \sup _{F \in C(M, \mathbb{R})}\left(\int F d v-P(\langle\xi, \Psi\rangle+F, \phi)+P(\langle\xi, \Psi\rangle, \phi)\right) \\
= & \sup _{F \in C(M, \mathbb{R})}\left(\int(F-\langle\xi, \Psi\rangle) d v-P(F, \phi)+P(\langle\xi, \Psi\rangle, \phi)\right) \\
= & \sup _{F \in C(M, \mathbb{R})}\left(\int F d v-P(F, \phi)\right)+P(\langle\xi, \Psi\rangle, \phi)-\int\langle\xi, \Psi\rangle d v \\
= & -\inf _{F \in C(M, \mathbb{R})}\left(P(F, \phi)-\int F d v\right)+P(\langle\xi, \Psi\rangle, \phi)-\int\langle\xi, \Psi\rangle d v .
\end{aligned}
$$

By Lemma 2.2, this is equal to

$$
-h_{\phi}(v)+P(\langle\xi, \Psi\rangle, \phi)-\int\langle\xi, \Psi\rangle d v>0,
$$

where the last inequality comes from the uniqueness of the equilibrium state $\mu_{\langle\xi, \Psi\rangle}$. Also by Lemma 2.2, the map on $\mathcal{M}(\phi)$ given by

$$
v \mapsto-h_{\phi}(v)+P(\langle\xi, \Psi\rangle, \phi)-\int\langle\xi, \Psi\rangle d v
$$

is lower semi-continuous and so we can conclude that $\rho+Q(0)>0$, as required. 
Proof of Theorem 9.2 Given a function $F \in C(M, \mathbb{R})$, choose $\delta>0$ and set $\mathcal{K}$ to be the compact set

$$
\mathcal{K}=\left\{v \in \mathcal{M}(\phi):\left|\int F d v-\int F d \mu_{\langle\xi, \Psi\rangle}\right| \geq \delta\right\} .
$$

Applying Theorem 9.6, we have

$$
\frac{1}{\Xi(T, \epsilon)} \sum_{\substack{\gamma \in \mathcal{P}(\phi): \\ T<l(\gamma) \leq T+\epsilon \\\langle\gamma\rangle=e}} \int F d \mu_{\gamma}=\frac{1}{\Xi(T, \epsilon)} \sum_{\substack{\gamma \in \mathcal{P}(\phi): \\ T<l(\gamma) \leq T+\epsilon \\\langle\gamma\rangle=e, \mu_{\gamma} \notin \mathcal{K}}} \int F d \mu_{\gamma}+O\left(e^{-\eta T}\right),
$$

for any $0<\eta<\rho_{\mathcal{K}}+P(\langle\xi, \Psi\rangle, \phi)$, so we only need to consider the limit of the first term on the Right Hand Side. We have

$$
\begin{aligned}
\frac{1}{\Xi(T, \epsilon)} \sum_{\substack{\gamma \in \mathcal{P}(\phi): \\
T<l(\gamma) \leq T+\epsilon \\
\langle\gamma\rangle=e, \mu_{\gamma} \notin \mathcal{K}}} \int F d \mu_{\gamma}=\left(1-O\left(e^{-\eta T}\right)\right) \int F d \mu_{\langle\xi, \Psi\rangle} \\
+\frac{1}{\Xi(T, \epsilon)} \sum_{\substack{\gamma \in \mathcal{P}(\phi): \\
T<l(\gamma) \leq T+\epsilon \\
\langle\gamma\rangle=e, \mu_{\gamma} \notin \mathcal{K}}}\left(\int F d \mu_{\gamma}-\int F d \mu_{\langle\xi, \Psi\rangle}\right)
\end{aligned}
$$

and therefore

$$
\limsup _{T \rightarrow \infty} \frac{1}{\Xi(T, \epsilon)} \sum_{\substack{\gamma \in \mathcal{P}(\phi): \\ T<l(\gamma) \leq T+\epsilon \\\langle\gamma\rangle=e, \mu_{\gamma} \notin \mathcal{K}}} \int F d \mu_{\gamma} \leq \int F d \mu\langle\xi, \Psi\rangle+\delta
$$

and

$$
\liminf _{T \rightarrow \infty} \frac{1}{\Xi(T, \epsilon)} \sum_{\substack{\gamma \in \mathcal{P}(\phi): \\ T<l(\gamma) \leq T+\epsilon \\\langle\gamma\rangle=e, \mu_{\gamma} \notin \mathcal{K}}} \int F d \mu_{\gamma} \geq \int F d \mu\langle\xi, \Psi\rangle-\delta
$$

Since $\delta>0$ is arbitrary, this completes the proof.

Open Access This article is licensed under a Creative Commons Attribution 4.0 International License, which permits use, sharing, adaptation, distribution and reproduction in any medium or format, as long as you give appropriate credit to the original author(s) and the source, provide a link to the Creative Commons licence, and indicate if changes were made. The images or other 
third party material in this article are included in the article's Creative Commons licence, unless indicated otherwise in a credit line to the material. If material is not included in the article's Creative Commons licence and your intended use is not permitted by statutory regulation or exceeds the permitted use, you will need to obtain permission directly from the copyright holder. To view a copy of this licence, visit http://creativecommons.org/licenses/by/4.0/.

\section{References}

1. Anantharaman, N.: Precise counting results for closed orbits of Anosov flows. Ann. Sci. École Norm. Sup. 33, 33-56 (2000)

2. Anosov, D.: Geodesic flows on closed Riemannian manifolds of negative curvature. In: Proceedings of the Steklov Institute of Mathematics 90: Translated from the Russian by S, p. 1969. Feder, American Mathematical Society, Providence, R.I (1967)

3. Barthelmé, T., Bonatti, C., Gogolev, A., Rodriguez-Hertz, F.: Anomalous Anosov flows revisited. Proc. London Math. Soc. https://doi.org/10.1112/plms.12321

4. Barthelmé, T., Fenley, S.: Counting orbits of Anosov flows in free homotopy classes. Comment. Math. Helvetici. 92, 641-714 (2017)

5. Bowen, R.: Symbolic dynamics for hyperbolic flows. Am. J. Math. 95, 429-460 (1973)

6. Bowen, R., Ruelle, D.: The ergodic theory of Axiom A flows. Invent. Math. 29, 181-202 (1975)

7. Brooks, R.: The fundamental group and the spectrum of the Laplacian. Comment. Math. Helvetici 56, 581-598 (1981)

8. Brooks, R.: The bottom of the spectrum of a Riemannian covering. J. Reine Angew. Math. 357, 101-114 (1985)

9. Coulon, R., Dal'Bo, F., Sambusetti, A.: Growth gap in hyperbolic groups and amenability. Geom. Funct. Anal. 28, 1260-1320 (2018)

10. Coulon, R., Dougall, R., Schapira, B., Tapie, S.: Twisted Patterson-Sullivan measures and applications to amenability and coverings, arXiv:1809.10881 [math.DG, math.DS] (2018)

11. Day, M.M.: Convolutions, means, and spectra. Ill. J. Math. 8, 100-111 (1964)

12. Denker, M., Urbanski, M.: On the existence of conformal measures. Trans. Am. Math. Soc. 328, 563-587 (1991)

13. Dougall, R.: Critical exponents of normal subgroups, the spectrum of group extended transfer operators, and Kazhdan distance. Adv. Math. 349, 316-347 (2019)

14. Dougall, R., Sharp, R.: Amenability, critical exponents of subgroups and growth of closed geodesics. Math. Ann. 365, 1359-1377 (2016)

15. Eberlein, P.: Geodesic flows on negatively curved manifolds I. Ann. Math. 95, 492-510 (1972)

16. Fenley, S.: Anosov flows in 3-manifolds. Ann. Math. 139, 79-115 (1994)

17. Fisher, T., Hasselblatt, B.: Hyperbolic flows. Zurich Lectures in Advanced Mathematics. European Mathematical Society, (2019)

18. Franks, J., Williams, R.: Anomalous Anosov flows. Global Theory of Dynamical Systems. In: Nitecki, Z., Robinson, C. (eds.) Lecture Notes in Mathematics, vol. 819, pp. 158-174. Springer-Verlag, Berlin, Heidelberg (1980)

19. Gurevič, B.: Topological entropy for denumerable Markov chains. Soviet Math. Dokl. 10, 911-915 (1969)

20. Jaerisch, J.: Group-extended Markov systems, amenability, and the Perron-Frobenius operator. Proc. Am. Math. Soc. 143, 289-300 (2015)

21. Katsuda, A., Sunada, T.: Homology and closed geodesics in a compact Riemann surface. Am. J. Math. 110, 145-156 (1988)

22. Katsuda, A., Sunada, T.: Closed orbits in homology classes. Inst. Hautes Études Sci. Publ. Math. 71, 5-32 (1990) 
23. Kesten, H.: Full Banach mean values on countable groups. Math. Scand. 7, 146-156 (1959)

24. Kitchens, B.: Symbolic Dynamics: One-sided, Two-sided and Countable State Markov Shifts. Springer-Verlag, Berlin-Heidelberg, New York (1998)

25. Kotani, M.: A note on asymptotic expansions for closed geodesics in homology classes. Math. Ann. 320, 507-529 (2001)

26. Lalley, S.: Closed geodesics in homology classes on surfaces of variable negative curvature. Duke Math. J. 58, 795-821 (1989)

27. Manning, A.: Axiom A diffeomorphisms have rational zeta functions. Bull. London Math. Soc. 3, 215-220 (1971)

28. Parry, W., Pollicott, M.: Zeta functions and the periodic orbit structure of hyperbolic dynamics. Astérisque 187(188), 1-268 (1990)

29. Paulin, F., Pollicott, M., Schapira, B.: Equilibrium states in negative curvature. Astérisque 373, 1-281 (2015)

30. Phillips, R., Sarnak, P.: Geodesics in homology classes. Duke Math. J. 55, 287-297 (1987)

31. Pollicott, M.: Homology and closed geodesics in a compact negatively curved surface. Am. J. Math. 113, 379-385 (1991)

32. Pollicott, M., Sharp, R.: Rates of recurrence for $\mathbb{Z}^{q}$ and $\mathbb{R}^{q}$ extensions of subshifts of finite type. J. London. Math. Soc. 49, 401-416 (1994)

33. Pollicott, M., Sharp, R.: Asymptotic expansions for closed orbits in homology classes. Geom. Dedicata 87, 123-160 (2001)

34. Ratner, M.: Markov partitions for Anosov flows on $n$-dimensional manifolds. Israel J. Math. 15, 92-114 (1973)

35. Roblin, T.: Un théorème de Fatou pour les densités conformes avec applications aux revêtements Galoisiens en courbure négative. Israel J. Math. 147, 333-357 (2005)

36. Ruelle, D.: Thermodynamic Formalism. Addison-Wesley, Reading, MA (1978)

37. Sarig, O.: Thermodynamic formalisms for countable Markov shifts. Ergodic Theory Dynam. Syst. 19, 1565-1595 (1999)

38. Savchenko, S.: Cohomological inequalities for finite topological Markov chains. Funct. Anal. Appl. 33, 236-238 (1999)

39. Sharp, R.: Closed orbits in homology classes for Anosov flows. Ergodic Theory Dynam. Sys. 13, 387-408 (1993)

40. Sharp, R.: A local limit theorem for closed geodesics and homology. Trans. Am. Math. Soc. 356, 4897-4908 (2004)

41. Sinai, Y.: Gibbs measures in ergodic theory. Russian Math. Surv. 27, 21-70 (1972)

42. Stadlbauer, M.: An extension of Kesten's criterion for amenability to topological Markov chains. Adv. Math. 235, 450-468 (2013)

43. Stadlbauer, M.: On conformal measures and harmonic functions for group extensions. In: Pacifico, M .J., Guarino, P. (eds.) In the Proceedings of "New Trends in One-Dimensional Dynamics", pp. 275-304. Springer Proceedings in Mathematics \& Statistics, Springer Nature, Switzerland (2019)

44. Walters, P.: An Introduction to Ergodic Theory, Graduate Texts in Mathematics 79. Springer-Verlag, New York-Heidelberg-Berlin (1982)

Publisher's Note Springer Nature remains neutral with regard to jurisdictional claims in published maps and institutional affiliations. 